This item was submitted to Loughborough's Institutional Repository (https://dspace.lboro.ac.uk/) by the author and is made available under the following Creative Commons Licence conditions.

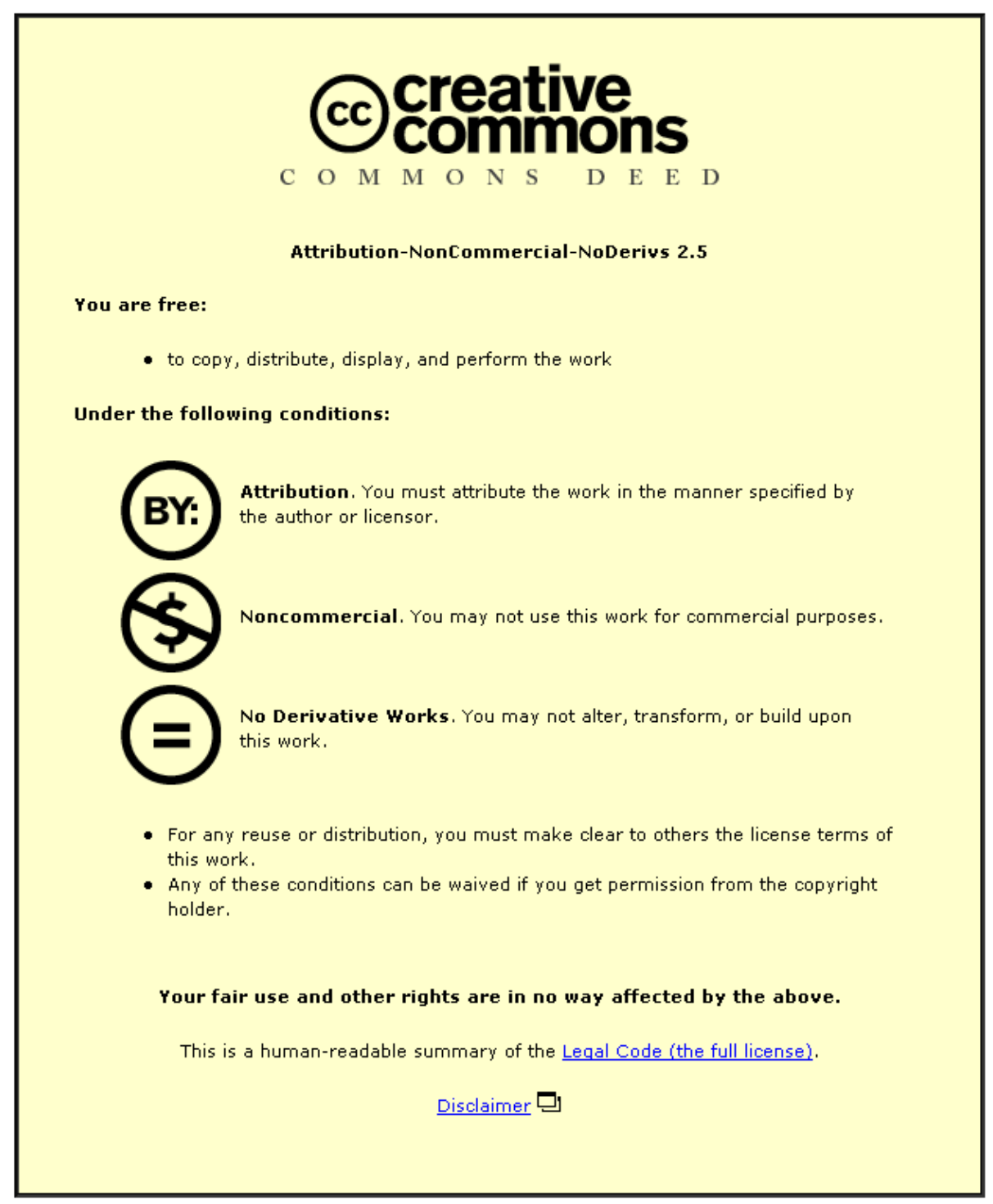

For the full text of this licence, please go to: http://creativecommons.org/licenses/by-nc-nd/2.5/ 


\title{
Convection in three-dimensional vibrofluidized granular beds
}

\author{
H. VISWANATHAN†, N. A. SHEIKH, R. D. WILDMAN \\ AND J. M. HUNTLEY \\ Wolfson School of Mechanical and Manufacturing Engineering, Loughborough University, \\ Loughborough, Leicestershire LE11 3TU, UK
}

(Received 25 March 2009; revised 15 April 2011; accepted 9 May 2011;

first published online 1 August 2011)

We study convective motion in vertically vibrated three-dimensional granular beds by comparing the predictions of a model based on a hydrodynamic description to Navier-Stokes order with experimental results obtained using positron emission particle tracking (PEPT). The three-dimensional conservation equations relating mass, momentum and energy are solved using the finite element (FE) method for a viscous vibrofluidized bed by using only observable system parameters such as particle number, size, mass and coefficients of restitution. The mean velocity profiles from the viscous model show reasonable agreement with the experimental results at relatively low altitudes for the range of experimental values studied, though the velocity fields at higher altitudes were systematically underestimated by the model. We confirm that the convection rolls are influenced by the sidewall coefficient of restitution and demonstrate the scaling relationships that operate, where increasing amplitude of vibration leads to a reduction in the angular velocity of the rolls.

Key words: fluidized beds, granular media, kinetic theory

\section{Introduction}

Granular materials, when subject to vertical vibration, exhibit a variety of phenomena which are non-uniform in space and time. Some of the commonly reported instabilities are convection, wave formation (shock \& standing waves) and clustering (Falcon et al. 1999). It has been known since Faraday (1831) that granular materials in a vibrating bed develop permanent convective rolls when the strength of the vibration exceeds a critical value. Unlike the well-known Rayleigh-Bénard thermal convection in fluids, however, the origin of granular convection has remained relatively unexplored since its discovery. Recent experiments using positron emission particle tracking (PEPT) (Wildman et al. 2000; Wildman, Huntley \& Parker 2001a; Wildman, Huntley \& Parker 2001b) have substantially aided our understanding of convection in vibrofluidized granular beds through visualization. Explanations for low vibration amplitude convection in granular materials have been proposed by a number of authors. Savage (1988) suggested that a form of acoustic streaming drove the convective rolls while Knight et al. (1996) have suggested that the nature of the collisions with the wall creates a ratcheting effect that causes the convection. The experimental results have been complemented by a description of convection that 
relies on an analysis of void probabilities near the walls (Shinbrot et al. 1997) and predicts many of the features observed by Knight et al. (1996).

Hayakawa, Yue \& Hong (1995) and Bourzutschky \& Miller (1995) have attempted to solve the conservation equations for dense, weakly vibrated beds using a numerical solution scheme, whilst convective instabilities of granular materials have also been investigated using two-dimensional traffic equations (Hong \& Yue 1998). These showed that bouncing solutions of two and four convection rolls could be observed and a change in convection pattern was detected by altering the aspect ratio of the system. Khain \& Meerson (2003) employed a Navier-Stokes like granular hydrodynamics with periodic boundaries to determine the threshold of thermal convection in terms of the inelasticity of the particles, using the Froude and Knudsen numbers. Their results indicated that convection instabilities are formed due to the presence of steeper temperature gradients that arise with increased numbers of particles or dissipation due to collisions.

Experimentally, free buoyancy-driven convection has been observed in threedimensional highly fluidized granular flows (Wildman et al. 2001a,b). The presence of density variations with negative temperature gradients were interpreted as the mechanism by which convection rolls were initiated. Similar behaviour has also been used to observe convection in a vibrofluidized bed containing two sizes of particles (Wildman \& Parker 2002).

Molecular dynamics simulations have shown that convection is expected to occur for a fully enclosed system when dissipation exceeds a threshold value (Ramírez, Risso \& Cordero 2000; Paolotti et al. 2004). In this study, the particle wall collisions were elastic, and thus, this convection was independent of the material properties of the wall. In the work by Sunthar \& Kumaran (2001), an event-driven simulation of a dilute vibrofluidized granular material in two dimensions was performed. Two phenomena, both showing inhomogeneity in the horizontal direction were observed. They are convection rolls similar to the Rayleigh-Bénard thermal convection in fluids (Chandrashekar 1981), and a clustering instability, where the bed spontaneously phase separates into coexisting dense and dilute regions. The convection acts to reduce the temperature gradient of the system; granular temperature differences were seen to reduce following the onset of convection in a study of the characterization of the stationary states of a dilute vibrofluidized granular bed. Detailed investigations by Sunthar \& Kumaran (2001) showed that these are different from the known instabilities in a vibrated granular medium. Characterization of these instabilities was carried out with a phase diagram using suitable parameters from the kinetic theory of vibrofluidized beds (Sunthar \& Kumaran 2001). Talbot \& Viot (2002) studied a threedimensional vibrofluidized bed using event-driven molecular dynamics simulations which provided good agreement with the experimental density, granular temperature and velocity field profiles from PEPT experiments. They demonstrated that the direction and intensity of the circulation are strong functions of the particle-wall restitution coefficient and also suggested modifications to the system to generate new buoyancy-driven convection patterns which were later observed by Wildman et al. (2005).

Eshuis et al. (2005) and Eshuis, van der Meer \& Alam (2010) have considered similar phenomena from the standpoint of an analogue to the Leidenfrost effect (Leidenfrost 1966; Meerson, Poschel \& Bromberg 2003). In this scenario, a highdensity region is supported from below by a highly energetic band of gas. At a critical shaking strength, this becomes unstable and local convection rolls are observed, even in an unbounded system. There are, then, two possible causes for convection. First, 
an instability in the bulk caused by, for example, clustering or inversion, can lead to spontaneous convection roll formation. Second, the instability can be imposed on the system through the boundary, which when inelastic causes curvature in the isotherms and drives convection.

Recently, Cordero, Ramírez \& Risso (2003) have numerically integrated the hydrodynamic equations to study convection in a bi-dimensional granular gas in a square box, subject to gravity. Three of the walls were adiabatic while the base behaved as if the system were in contact with a 'heat-bath'. The four walls were treated as shear-free. The solutions predict buoyancy-driven convection triggered by a dynamically created 'temperature gradient'. However, in actual experiments, the energy source is typically a vibrating base unlike the thermal boundary condition as treated above. Also, an adiabatic boundary condition at the sidewalls is not equivalent to the boundary conditions employed in experiments, as wall effects are highly significant in describing convection (Wildman et al. 2001a). A number of researchers have derived formulae using kinetic theory for the transport coefficients in the hard sphere case (which at the simplest level are the granular equivalents to the Fourier Law), the stress tensor and the diffusion laws (Jenkins \& Savage 1983; Jenkins \& Richman 1985; Jenkins 1999). More recent derivations have shown the presence of a term proportional to the gradient of the number density in the heat constitutive relations, in addition to the usual temperature gradient (Brey et al. 1998; Sela \& Goldhirsch 1998; Garzó \& Dufty 1999). Martin, Huntley \& Wildman (2005) developed radially averaged models based on different kinetic-theory-based models which predicted both the granular temperature and the packing fraction as a function of altitude for a vibrofluidized bed. This included the effect of the dissipation at the walls in a through-thickness averaged way, but was unable to predict the radial variation. Subsequently, Viswanathan et al. (2006) took a similar approach by extending the analysis of Martin et al. (2005) to the axisymmetric (3D) case and solved the equations with the variables retained as two-dimensional quantities, allowing the granular temperature and packing fraction to be predicted as a function of both height and radius. However, the effect of viscosity was neglected in both of these models and they were therefore incapable of predicting the steady-state convection patterns that are observed experimentally (Wildman et al. 2001a).

In this paper, a hydrodynamic model based on the kinetic theory method is described and solved numerically to predict the convection behaviour of particles in a vibro-fluidized bed. The fundamental viscous hydrodynamic equations are employed with the appropriate closures derived using an approximation to the simplest type of flow: steady state with binary and instantaneous collisions. Subsequently, results are presented and compared to both numerical results from the inviscid model by Viswanathan et al. (2006) and experimental results obtained using PEPT (Wildman et al. 2001a, b).

\section{System description and hydrodynamic viscous model}

A cylindrical system of radius $R$ and height $L$ containing $N$ particles each with a diameter $d$ and mass $m$ was used to model the real-life vibro-fluidized granular system shown in figure 1, in which the base was vibrated sinusoidally at an angular frequency $\omega$ and amplitude $A_{0}$ using an electrodynamic shaker. The modelling technique involves solving the hydrodynamic equations for a dissipative fluid closed with constitutive relations appropriate for inelastic collisions between particles. To achieve this closure, we use the constitutive equations derived for frictionless (smooth), nearly elastic hard 


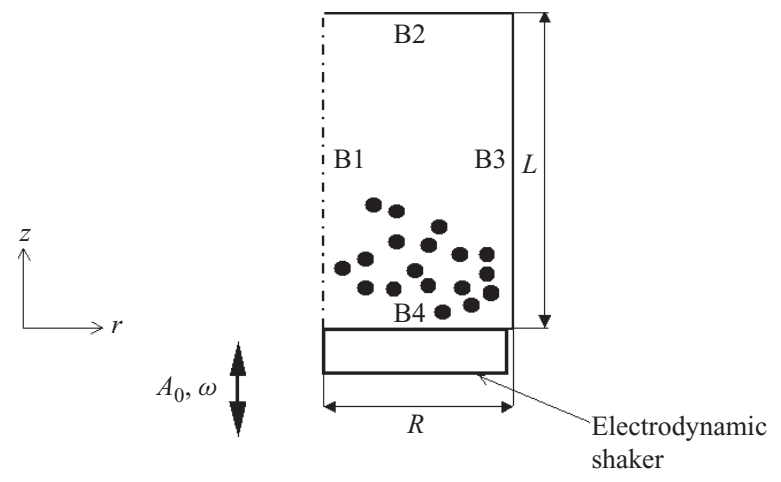

FIgURE 1. Axisymmetric solution domain. Boundary definitions are provided in the text.

spheres using Grad's 13-moment method for the solution of the inelastic EnskogBoltzmann equation (Jenkins \& Savage 1983; Jenkins \& Richman 1985; Jenkins 1999) and the derivation of Garzó \& Dufty (1999) using the revised Enskog Theory. These hydrodynamic equations describe the basic fields such as granular temperature, number density (packing fraction) and mean velocity field vector.

\subsection{Granular temperature}

In this work, we define the granular temperature in terms of the mean-squared fluctuating velocity, such that

$$
T=\frac{1}{3} m\left\langle c^{2}\right\rangle
$$

where $c$ is the speed of a particle about the mean velocity of the flow and \langle\rangle denotes an ensemble average. In a granular flow, equipartition does not necessarily hold (Feitosa \& Menon 2002; Wildman \& Parker 2002) and the stress tensor may be anisotropic. In this case, the granular temperatures in the $r, \theta$ and $z$ directions are given by

$$
\begin{gathered}
T_{r} \equiv m\left\langle u^{2}\right\rangle, \\
T_{\theta} \equiv m\left\langle v^{2}\right\rangle, \\
T_{z} \equiv m\left\langle w^{2}\right\rangle,
\end{gathered}
$$

where $u, v$ and $w$ are the velocity components of the particles in the respective directions.

\subsection{Hydrodynamic equations}

By considering the balance of mass, momentum and kinetic energy, it is possible to derive relations similar to the conservation equations for thermal systems (Landau \& Lifshitz 1987). These are, respectively,

$$
\begin{gathered}
\frac{\partial n}{\partial t}+\nabla \cdot(n \boldsymbol{u})=0 \\
n\left(\frac{\partial \boldsymbol{u}}{\partial t}+\boldsymbol{u} \cdot \nabla \boldsymbol{u}\right)=\nabla \cdot \boldsymbol{P}_{i j}-n g \hat{z}, \\
\frac{3}{2} n\left(\frac{\partial T}{\partial t}+\boldsymbol{u} \cdot \nabla T\right)=-\nabla \cdot \boldsymbol{J}+\boldsymbol{P}_{i j}: \underline{\boldsymbol{E}}-\gamma .
\end{gathered}
$$


where $t$ is time, $\boldsymbol{P}_{i j}$ is the stress tensor, vector $\boldsymbol{J}$ is the flux of the fluctuation energy, $\underline{\boldsymbol{E}}$ is the symmetric part of the velocity gradients, $n$ is the number density, $\gamma$ is the collisional rate of dissipation of the fluctuation energy per unit volume and $g$ is the acceleration due to gravity.

The components of the symmetrized velocity gradient tensor $\underline{\boldsymbol{E}}$ are given by (Landau \& Lifshitz 1987)

$$
E_{i j}=\frac{1}{2}\left(\partial_{j} u_{i}+\partial_{i} u_{j}\right) .
$$

The components of the stress tensor $\boldsymbol{P}_{i j}$ are split into two components $\boldsymbol{P}_{i j}=\boldsymbol{P}_{i j}^{0}+\boldsymbol{P}_{i j}^{1}$, where $\boldsymbol{P}_{i j}$ is related to hydrostatic pressure $P$ as

$$
\begin{gathered}
\boldsymbol{P}_{i j}^{0}=-P \delta_{i j} \\
\boldsymbol{P}_{i j}^{1}=\left(\left(\lambda-\frac{2}{3} \mu\right) E_{k k}\right) \delta_{i j}+2 \mu E_{i j},
\end{gathered}
$$

and the heat flux is given by

$$
\boldsymbol{J}=-\kappa \nabla T-\mu_{e} \nabla n,
$$

where $\kappa$ is the thermal conductivity and $\mu_{e}$ is additional transport coefficient due to inelasticity of granular media. In the following sections, two constitutive relationships are presented.

\subsection{Transport coefficients of Jenkins}

Thermal conductivity $\kappa$ is given by Jenkins (1999) as

$$
\kappa=\frac{4 M G P d}{\sqrt{\pi m}(1+4 G) T^{1 / 2}},
$$

where

$$
M=1+\frac{9 \pi}{32}\left(1+\frac{5}{12 G}\right)^{2}
$$

and

$$
G=\eta g_{0}
$$

where $\eta$ is the packing fraction. The radial distribution function at contact, $g_{0}$, is given by Carnahan \& Starling (1969)

$$
g_{0}=\frac{(2-\eta)}{2(1-\eta)^{3}} .
$$

In this case, the inelasticity coefficient is zero, i.e. $\mu_{e}=0$. The expressions for bulk dissipation, bulk viscosity and shear viscosity are given by Jenkins (1999), such that the bulk dissipation rate is

$$
\gamma=\frac{24}{\sqrt{\pi m}}(1-e) \frac{P}{1+4 G} G \frac{T^{1 / 2}}{d} .
$$

The bulk viscosity is given by

$$
\lambda=\frac{8}{3 \sqrt{\pi}} n d G T^{1 / 2},
$$

and the shear viscosity is

$$
\mu=\frac{\sqrt{\pi}}{6} n d T^{1 / 2}\left[\frac{5}{16 G}+1+\frac{4}{5}\left(1+\frac{12}{\pi}\right) G\right],
$$


where $e$ is the particle-particle coefficient of restitution (Jenkins 1999). The relationship between $n$ and the packing fraction, $\eta$, is given by

$$
n=\frac{6 \eta}{\pi d^{3}} \text {. }
$$

The second set of constitutive relationships use the dense granular gas inelastic relationships developed by Garzo \& Dufty (1999).

\subsection{Transport coefficients of Garzo and Dufty}

The transport coefficients are reduced to non-dimensional form using $\kappa_{0}$ and $\eta_{0}$ as follows:

$$
\begin{aligned}
\kappa^{*} & =\frac{\kappa}{\kappa_{0}}, \\
\mu^{*} & =\frac{\mu}{\eta_{0}}, \\
\lambda^{*} & =\frac{\lambda}{\eta_{0}}, \\
\mu_{e}^{*} & =\frac{\eta \mu_{e}}{T \kappa_{0}},
\end{aligned}
$$

where $\kappa_{0}=15 \eta_{0} / 4 m$ and $\eta_{0}=\left(5 m / 16 d^{2}\right)(\sqrt{T / \pi})$ are the dilute limit values of thermal conductivity and shear viscosity, respectively. The cooling rate $\gamma$ is reduced as $\gamma^{*}=\gamma / \nu_{0}$, with $v_{0}$ the characteristic collision frequency given by

$$
v_{0}=\frac{\rho T}{\eta_{0}} \text {. }
$$

The coefficients for the transport of bulk viscosity, shear viscosity and cooling rate are given as

$$
\lambda^{*}=\frac{32}{45} \pi \eta G(1+e)\left(1-\frac{1}{32} c^{*}\right)
$$

and

$$
\mu^{*}=\mu^{k}\left[1+\frac{2}{15} \pi G(1+e)\right]+\frac{3}{5} \lambda^{*},
$$

where the kinetic parts, $\mu^{k}$ and $c^{*}$, are given by

$$
\mu^{k}=\frac{1}{\left(v_{\eta}-\frac{1}{2} \gamma^{(0)}\right)}\left[1-\frac{1}{15}(1+e)(1-3 e) \pi G\right]
$$

and

$$
c^{*}=32(1-e)\left(1-2 e^{2}\right)\left[81-17 e+30 e^{2}(1-e)\right]^{-1}
$$

with

$$
\begin{gathered}
v_{\eta}=\frac{2-\eta}{2(1-\eta)^{3}}\left[1-\frac{1}{4}(1-e)^{2}\right]\left[1-\frac{1}{64} c^{*}\right], \\
\gamma^{(0)}=\frac{5}{12} \frac{2-\eta}{2(1-\eta)^{3}}\left(1-e^{2}\right)\left(1+\frac{3}{32} c^{*}\right) .
\end{gathered}
$$


The constitutive coefficients for $\kappa$ and $\mu$ are given by

$$
\kappa^{*}=\kappa^{k}\left[1+\frac{1}{5} \pi G(1+e)\right]+\frac{64}{225} \pi \eta G(1+e)\left(1+\frac{7}{32} c^{*}\right),
$$

where $\kappa^{k}$ is given by

$$
\begin{aligned}
\kappa^{k}=\frac{2}{3\left(v_{k}-2 \gamma^{(0)}\right)}\left[1+\frac{1}{2}(1\right. & \left.+p^{*}\right) c^{*}+\frac{\pi G}{10}(1+e)^{2} \\
& \left.\times\left\{2 e-1+\left[\frac{1}{2}(1+e)-\frac{5}{3(1+e)}\right] c^{*}\right\}\right]
\end{aligned}
$$

and $p^{*}$ and $v_{k}$ are given by

$$
p^{*}=1+\frac{1+\alpha}{3} \pi G
$$

and

$$
v_{k}=\frac{1}{3}(1+e) \frac{2-\eta}{2(1-\eta)^{3}}\left[1+\frac{33}{16}(1-e)+\frac{19-3 e}{1024} c^{*}\right] .
$$

The inelastic transport coefficient is given by

$$
\mu_{e}^{*}=\mu_{e}^{k}\left[1+\frac{1}{5} \pi G(1+e)\right]
$$

where the kinetic part $\mu_{e}^{k}$ is

$$
\mu_{e}^{k}=\frac{2}{\left(2 \nu_{k}-3 \gamma^{(0)}\right)}\left\{\begin{array}{l}
\left(1+\eta \partial_{\eta} \ln g_{0}\right) \gamma^{(0)} \kappa^{k}+\frac{p^{*}}{3}\left(1+\eta \partial_{\eta} \ln p^{*}\right) c^{*} \\
-\frac{2}{15} \pi G\left(1+\frac{1}{2} \eta \partial_{\eta} \ln g_{0}\right)^{2}(1+e)\left\{e(1-e)+\frac{1}{4}\left[\frac{4}{3}+e(1-e)\right] c^{*}\right\}
\end{array}\right\} .
$$

The full form of the cooling rate coefficient is given by $\gamma^{*}=\gamma^{(0)}+\gamma^{(1)}$, where $\gamma^{(1)}$ is

$$
\gamma^{(1)}=\left[-\frac{1}{v_{0}}(1-e)\left(p^{*}-1\right)+\frac{5}{32}\left(1-e^{2}\right)\left(1+\frac{3}{64} c^{*}\right) g_{0} c_{D}\right] \nabla \boldsymbol{u} .
$$

Here, $c_{D}$ is given by

$$
c_{D}=\frac{1}{v_{0}}\left[\frac{1}{2} \gamma^{(0)}+v_{r}+\frac{5 c^{*}}{64}\left(1+\frac{3}{64} c^{*}\right) g_{0}\left(1-e^{2}\right)\right]^{-1}\left[\frac{2}{45} \alpha \pi G+\left(p^{*}+1\right)\left(\frac{2}{3}-e\right) c^{*}\right],
$$

where $v_{r}$ and $\alpha$ are given by

$$
\begin{aligned}
v_{r} & =\frac{1+e}{48} g_{0}\left(128-96 e+15 e^{2}-15 e^{3}\right)+\frac{c^{*}}{64}\left(15 e^{3}-15 e^{2}+498 e-434\right), \\
\alpha & =\frac{3}{8}\left[(1-e)\left(5 e^{2}+4 e-1\right)+\frac{c^{*}}{12}\left(159 e+3 e^{2}-19 e-15 e^{3}\right)\right](1+e) .
\end{aligned}
$$

\subsection{Equation of state}

The moderately dense phase equation of state suggested by Garzo \& Dufty (1999)

$$
P=\eta T\left(1+\frac{1+\alpha}{3} \pi G\right)
$$

is used throughout this work. 

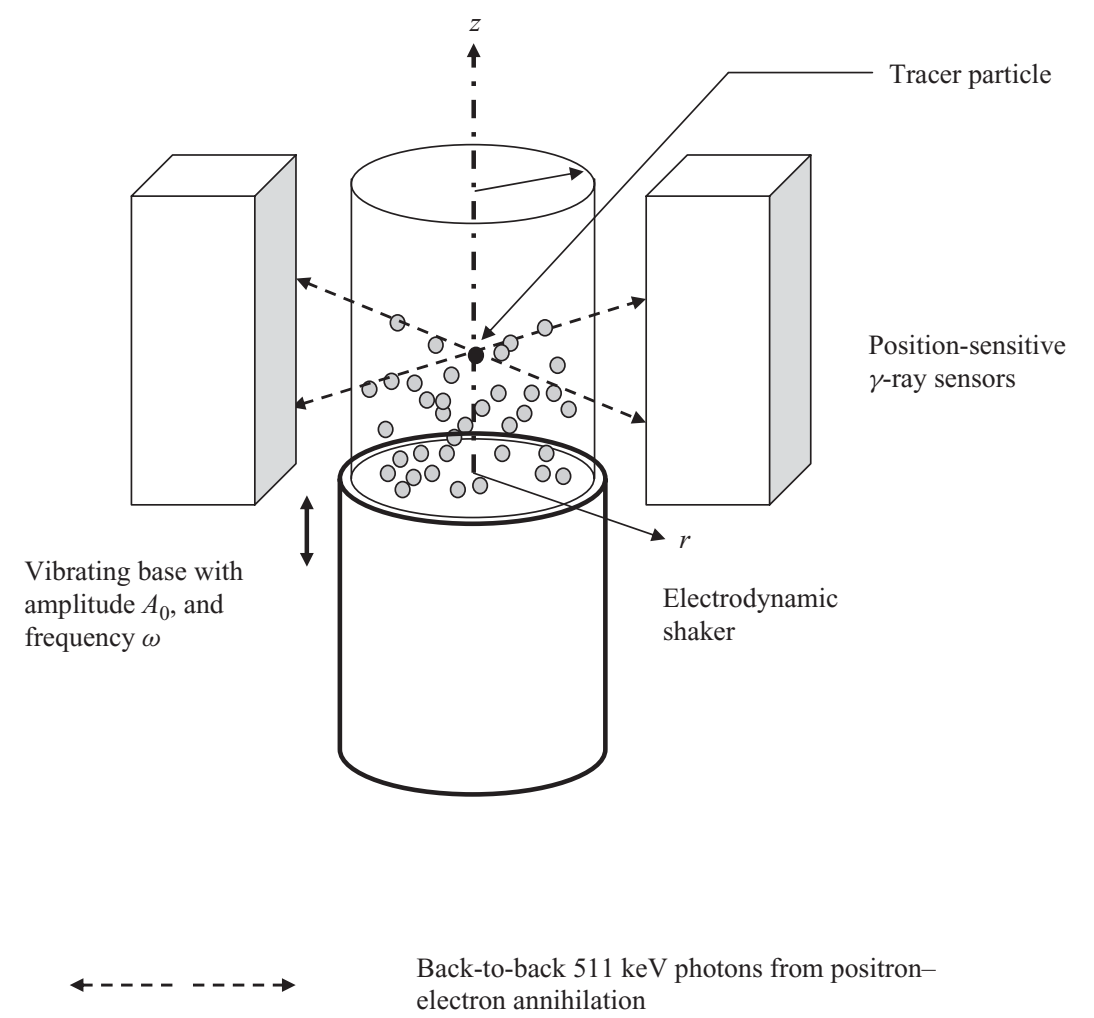

FIGURE 2. Schematic representation of a $3 \mathrm{D}$ vibrated bed.

\subsection{Boundary conditions}

The required set of boundary conditions for this problem is deduced from symmetry arguments, energy balance equations and a pressure condition along the four boundaries B1-B4 surrounding the domain of interest as defined in figure 1. The symmetry arguments and energy balance equations are expressed here in terms of the granular temperature $T$, the radial and axial components of the mean velocity field ( $u$ and $v$, respectively) or the gradients of these quantities on B1-B4. In addition, the pressure $P$ needs to be specified at the base. This may be done by specifying the pressure at the base in terms of the total mass of particles in the system.

Taking B1 (i.e. the axis of the cylinder, $r=0$ ) first, the axial symmetry of the problem allows the gradients of the temperature and of the components of the mean velocity fields to be specified as follows:

$$
\begin{gathered}
\frac{\partial T}{\partial r}=0, \\
\frac{\partial u}{\partial r}=0, \\
\frac{\partial v}{\partial r}=0 .
\end{gathered}
$$

The base of the cell (boundary B4 in figure 1) provides the energy input to the system. Detailed analysis on vibrating boundaries has been performed by a number of 
researchers, ranging from a consideration of particle trajectories near a base vibrating with a sawtooth profile (Warr \& Huntley 1995) to generalized profiles (Kumaran 1998) and bumpy boundaries (Richman 1993). Here, we adopt the expression derived by Richman (1993). He considered collisions with a moving bumpy boundary undergoing random vibrations along three perpendicular directions. In the limit of a smooth flat wall, the in-plane vibration components do not contribute to the energy transfer. The energy flux for a base moving with a mean square velocity of $\bar{V}_{b}^{2}$ only in the vertical direction is therefore identical to that for a base moving with a mean square velocity of $\bar{V}_{b}^{2}$ along each axis. The magnitude of the resulting energy flux at the base is then given by

$$
J(r, 0)=\left(\frac{2}{\pi}\right)^{1 / 2} 2 \rho G\left[2 \bar{V}_{b}^{2}\left(\frac{T}{m}+\bar{V}_{b}^{2}\right)^{1 / 2}-\left(1-e_{b}\right)\left(\frac{T}{m}+\bar{V}_{b}^{2}\right)^{3 / 2}\right] .
$$

The sidewall (B3, figure 1) is treated in a similar fashion to the base, such that

$$
J(r, R)=\left(\frac{2}{\pi}\right)^{1 / 2} 2 \rho G\left[2 \bar{V}_{w}^{2}\left(\frac{T}{m}+\bar{V}_{w}^{2}\right)^{1 / 2}-\left(1-e_{w}\right)\left(\frac{T}{m}+\bar{V}_{w}^{2}\right)^{3 / 2}\right],
$$

where $\bar{V}_{w}^{2}$ is the out of plane mean squared velocity component of the wall. Since there is no significant horizontal motion associated with the sidewalls in our experiments, we set $\bar{V}_{w}^{2}$ equal to zero. The quantities $e_{b}$ and $e_{w}$ represent the particle-base and particle-wall coefficients of restitution, respectively. At higher altitudes, collisions become increasingly infrequent and so in the limit $z \rightarrow \infty$ the heat flux through the upper boundary tends to zero. Experimentally, one observes the packing fraction at large heights to decay exponentially with $z$ so the above requirement is also a good approximation for a container of finite height provided it is sufficiently tall. Therefore, for the boundary B2, we write

$$
J(r, L)=0 .
$$

Here, $L$ is chosen to be 30 particle diameters, in line with the height of the experimental cell $(\S 4)$.

The base, sidewall and upper boundaries are impenetrable. Moreover, the walls are assumed to be smooth with negligible frictional forces, and therefore, the tangential stress at the wall vanishes. Hence, we employ the impenetrable boundary conditions, with slip, to specify the conditions for $u$ and $v$ on these boundaries (Getling 1998)

$$
\begin{gathered}
\boldsymbol{n} \cdot \boldsymbol{u}=0, \\
\boldsymbol{t} \cdot \boldsymbol{K}=0,
\end{gathered}
$$

where $\boldsymbol{u}$ is the velocity vector, $\boldsymbol{n}$ is the outward normal vector on the boundary, $\boldsymbol{t}$ represents a tangential vector in directions orthogonal to $\boldsymbol{n}$, and $\boldsymbol{K}$ is the viscous boundary force per unit area given by

$$
\boldsymbol{K}=\left(-P \boldsymbol{I}+\mu\left(\nabla \boldsymbol{u}+(\nabla \boldsymbol{u})^{\mathrm{T}}\right)+\left(\frac{2 \mu}{3}-\lambda\right)(\nabla \boldsymbol{u}) \boldsymbol{I}\right) \boldsymbol{n},
$$

where I represents the identity or unit diagonal matrix.

\section{Numerical solution method}

The Navier-Stokes-like equations $((2.3)-(2.5))$ along with the boundary conditions $((2.40 \mathrm{a})-(2.44 c))$ were solved numerically as a steady-state problem using a 
commercially available finite-element (FE) package, COMSOL Multiphysics (version 3.2), interfaced with MATLAB (version 6.5). The mass and momentum conservation equations (2.3) and (2.4) were implemented in 'Non-Isothermal mode' in the Chemical Engineering-Momentum balance module. The energy equation (2.5) was implemented in 'Convection and Conduction mode' in the Chemical Engineering-Energy balance module. The equation of state (2.39) was implemented as an algebraic equation using the general form in the PDE module. These equations were solved using a triangular mesh with different mesh qualities such as (i) 200 elements, 30 boundary elements and 1293 degrees of freedom, (ii) 438 elements, 44 boundary elements and 1842 degrees of freedom, (iii) 682 elements, 56 boundary elements and 4263 degrees of freedom, (iv) 1456 elements, 82 boundary elements and 8985 degrees of freedom. It was observed that the numerical error was small $(<1 \%)$ with mesh quality (ii) and any refinements above it (meshes (iii) and (iv)). Hence, the mesh quality (ii) was chosen to compute the numerical results presented in this paper. A stationary nonlinear solver was used with a generalized mean residual (GMRES) linear system solver and incomplete LU preconditioner with a drop tolerance of $10^{-6}$. We also used the 'Elimination' constraint handling method and 'Orthonormal' null-space function to obtain a solution. Using a previously obtained solution as an initial estimate, results for any set of parameters (e.g. different $N$ or $A_{0}$ values) can be obtained by iteration until convergence to a relative error of the order of $10^{-9}$ is reached.

\section{Experimental details}

For comparison of the numerical results with experiment, we used the PEPT technique. A $145 \mathrm{~mm}$ diameter polymethyl methacrylate (PMMA) cylindrical cell (height $300 \mathrm{~mm}$ ) containing one layer of particles (700 glass beads) each with a diameter of $5 \mathrm{~mm}$ was vibrated, thus fluidizing the granular beds. The coefficients of restitution were measured using high-speed photography as being $0.91 \pm 0.02$ for a glass-glass collision (particle-particle, particle-base) and $0.68 \pm 0.04$ for a glassPMMA collision (particle-wall). The cylinder was attached to a Ling dynamic systems vibrator, which vibrated the system at a frequency of $50 \mathrm{~Hz}$ and amplitude of up to $1.54 \mathrm{~mm}$ with a sinusoidal waveform (figure 2). PEPT involves labelling a single tracer particle with radioactive nuclei. These nuclei decay by positron emission, and the positrons emitted rapidly annihilate with a local electron, producing two gamma rays travelling in opposite directions. These gamma rays are detected by two position sensitive gamma ray detectors placed either side of the system and an accurate location can be determined from detection of a small number of back-to-back pairs (figure 2). Coincident detection of two gamma rays in a pair of position-sensitive detectors defines a line passing close to the point of emission. However, a number of events are used to correct for scattering within the system. Through triangulation of successive location events, the position of a tracer particle can be located. As the tracer particle used here was identical to all the others, apart from its radioactivity, the trajectory obtained is representative of all the particles, and can be used to calculate the system distributions (Wildman et al. 2000; Wildman et al. 2001a,b).

The experimental details regarding this technique, its application to the measurement of granular flows and the data analysis procedure used to calculate the granular temperature and the packing fraction have been discussed previously and the reader is directed to references (Wildman et al. 2000, 2001a,b, 2002, 2005) for more details. The same procedure is employed here, with the extension of the measurements to variation in both the $z$ and $r$ directions. 


$\begin{array}{ll}\text { Dimensionless variable } & \text { Dimensional relationship } \\ T^{*} & T / m g d \\ P^{*} & P \pi d^{2} / 6 m g \\ \boldsymbol{u}^{*} & \boldsymbol{u} / \sqrt{ }(g d) \\ V_{b}^{*} & V_{b} / \sqrt{ }(g d) \\ V_{v}^{*} & V_{v} / \sqrt{ }(g d) \\ z^{*} & z / d \\ r^{*} & r / d \\ \Omega^{*} & \Omega / \sqrt{ }(g / d) \\ A_{0}^{*} & A_{0} / d\end{array}$

TABLE 1. Relationship between the dimensional and dimensionless variables.

\section{Results and discussion}

\subsection{Validation of the model}

For the purpose of providing a partial validation of the numerical results, the predictions of granular temperature and packing fraction profiles were compared against the previously validated 1D results obtained from an inviscid model (Viswanathan et al. 2006), which were, in turn, shown to be in excellent agreement with the results of Martin et al. (2005), for the case of elastic sidewalls. The calculations are presented in non-dimensional units with conversions provided in table 1. Figure 3 shows a comparison between the calculated non-dimensional granular temperature and packing fraction fields from the inviscid model obtained by Viswanathan et al. (2006), and the two models described in the previous section (along the line $r^{*}=0$ ) for various $N$ values when the non-dimensional root-mean-square base velocity $V_{b}^{*}=1.54$ and the particle-wall restitution coefficient is set to unity. In this limit, the magnitudes of the mean non-dimensional velocity fields were of the order of $10^{-6}$ and so one would expect an excellent agreement between the models near the base. In figure $3(a)$, when the dimensionless granular temperature is plotted as a function of height, the granular temperatures decay asymptotically, until about $4 \sigma$, where the temperature in the Garzo-Dufty model reaches a minimum and then increases. Similarly, the packing fraction predictions are compared in figure $3(b)$ and as expected show a small 'hump' near the base for relatively large values of $N$. The difference between the results of the models at $z^{*}<4 \sigma$ was around $(<0.5 \%)$ in $T^{*}$ and in $\eta$, indicating excellent agreement between the two.

\subsection{Comparison with experiment}

Figures 4 and $5\left(V_{b}^{*}=1.14\right.$ and 1.54 , respectively) show the predicted granular temperature fields and their experimental counterparts for a situation where sidewall dissipation is turned on. In each case, the vertical cross-sections of the experimental granular temperatures in the vertical $\left(T_{z}^{*}\right)$ and horizontal directions $\left(T_{r}^{*}\right)$, and the total granular temperature $\left(T^{*}\right)$ are plotted at different radial positions. These are compared to the results from the inviscid model from Viswanathan et al. (2006) and the two presented in this paper. In each case, the granular temperature predicted by the viscous model is seen to be lower than that from the inviscid model. This suggests that despite the creation of fluctuations due to shear work being done during the convective motion, the inclusion of viscous stress creates the conditions for more collisional dissipation within the bulk system. 

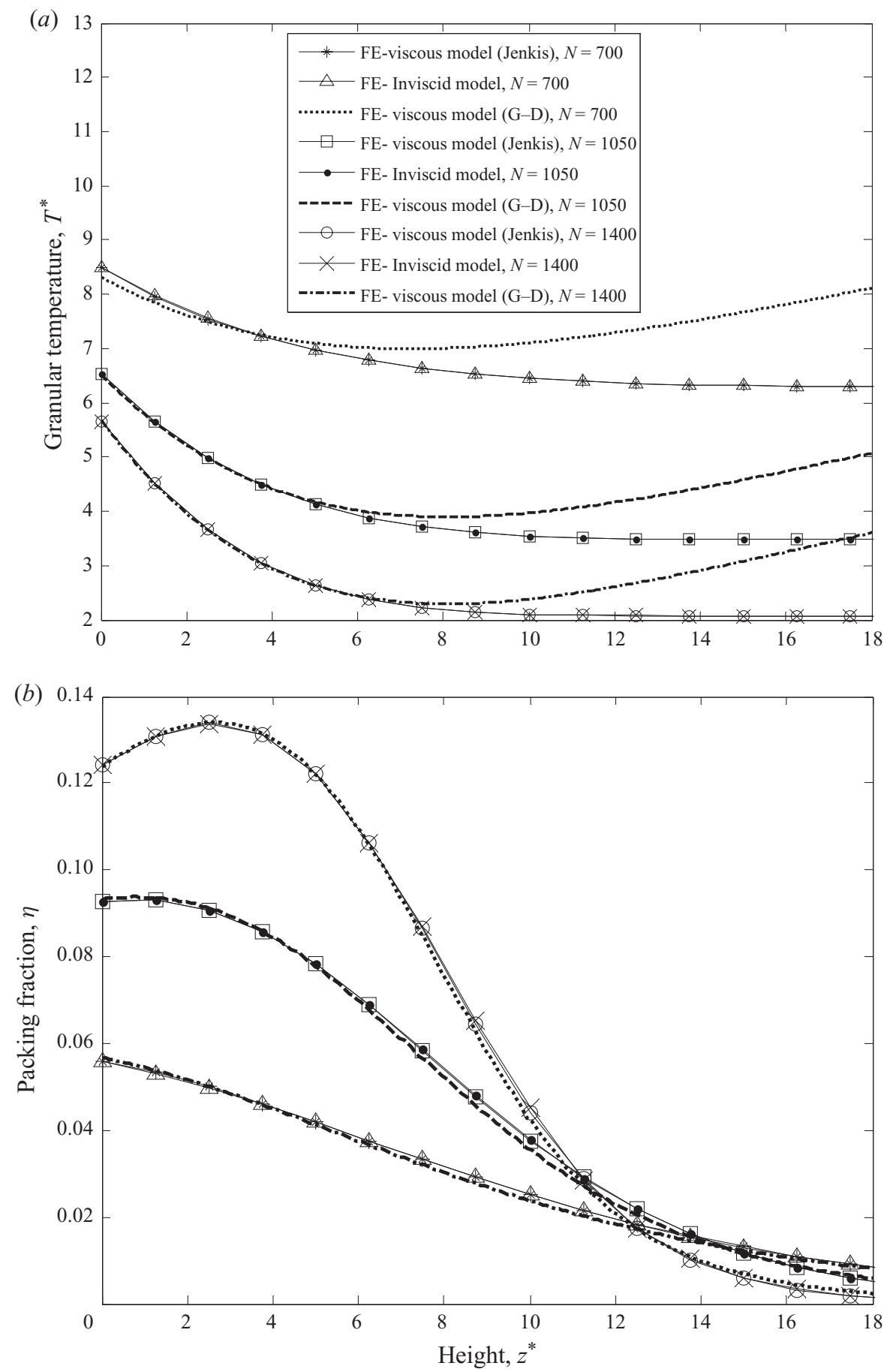

FIGURE 3. Comparison of $(a)$ dimensionless granular temperature and $(b)$ packing fraction profiles, for various values of $N$, between the inviscid model (Viswanathan) and the viscous model (Jenkins) for the case $V_{b}^{*}=1.54, e=e_{b}=0.91$ and $e_{w}=1$. 

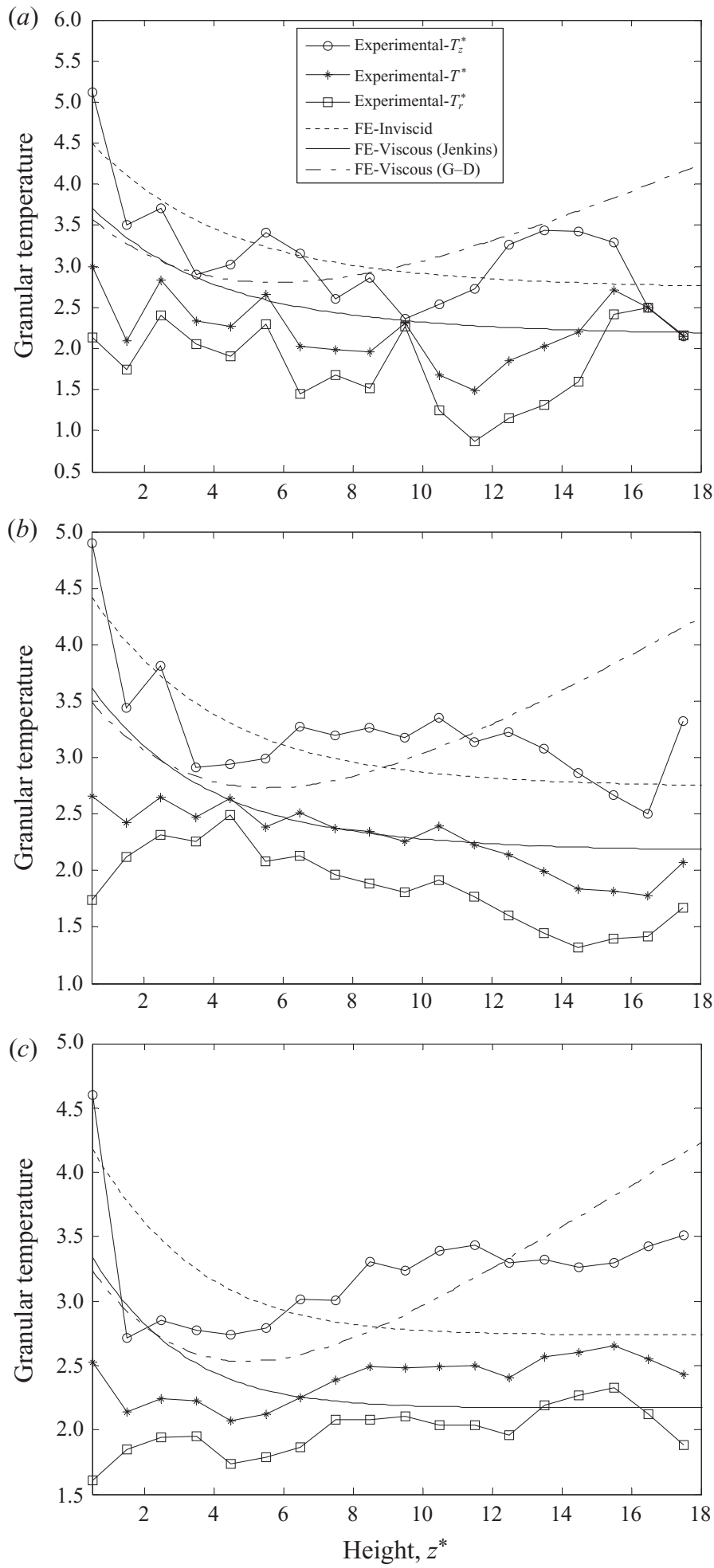

FIGURE 4. Experimental results and predictions from three hydrodynamic models for the vertical cross-sections of dimensionless granular temperature fields at the dimensionless radial positions $(a) r^{*}=0.72,(b) r^{*}=5.07$ and $(c) r^{*}=9.42$ for the case $e_{w}=0.68, V_{b}^{*}=1.14, N=700$ and $e=e_{b}=0.91$. 

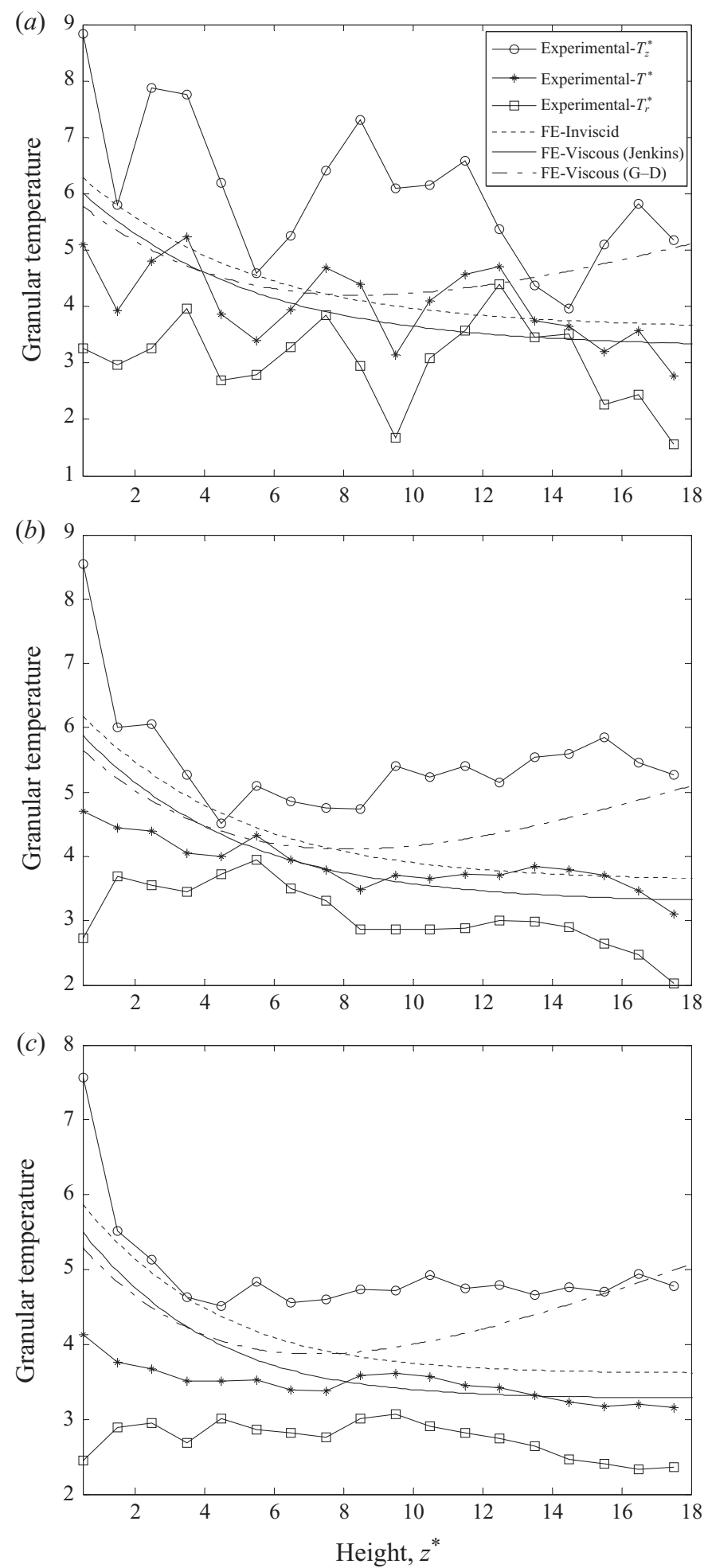

FIGURE 5. Experimental results and predictions from three hydrodynamic models for the vertical cross-sections of dimensionless granular temperature fields at the dimensionless radial positions $(a) r^{*}=0.72,(b) r^{*}=5.07$ and $(c) r^{*}=9.42$ for the case $e_{w}=0.68, V_{b}^{*}=1.54, N=700$ and $e=e_{b}=0.91$. 
The experimental results show that the fluctuations of the $z$-component of velocity, $T_{z}^{*}$, decay close to the base, but there is a suggestion that at high altitudes it increases with altitude. The results for the $r$-component, $T_{r}^{*}$, show a small increase with height as the energy is transferred from the $z$ direction to the $r$, but then show a slow decay with increasing height and distance from the axis. At the lower vibration amplitude $V_{b}^{*}=1.14$, closer to the axis (figure $4 a$ ), the $z$-component of temperature $\left(T_{z}^{*}\right)$ is seen to be in reasonable agreement with the model of Jenkins, and that of Garzo and Dufty, although the same degree of upturn is not seen. As has already been noted however (Martin et al. 2005), we do not anticipate agreement at large distances from the base. Further away from the axis, the agreement between the total temperature $T^{*}$ and temperature predicted by the viscous model gets better (figures $4 b$ and $4 c$ ). The standard error associated with the granular temperature measurements is typically below 1 at heights up until $z^{*} \sim 4 \sigma$, which then grows to be in excess of 10 at altitudes above $z^{*}=18 \sigma$, suggesting that the fluctuations at upper altitudes cannot be considered significant.

In figures 6 and 7, the packing fraction profiles obtained from the experimental data are compared against the inviscid and the viscous model results for $V_{b}^{*}=1.14$ and 1.54, respectively. Both the theoretical predictions show a monotonically decreasing packing fraction with height at each radial position. In the radial direction, the experimental packing fraction values increase as the wall is approached, which may be interpreted as being due to the relatively high grain-wall energy losses. This feature is reproduced by both the viscous and the inviscid models for both of the amplitudes. In all the cases, the viscous model is seen to predict higher packing fractions closer to the base than the inviscid model for the same parameters, and both models predict a higher packing fraction than the experimental results in the axial regions (figures $6 a$ and $7 a$ ). The experimental packing fraction profiles further away from the axis tend to agree better with the results of the viscous models as the base amplitude is increased (figures $6 c$ and $7 c$ ). Neither the viscous nor the inviscid model predictions from Viswanathan et al. (2006) completely capture the 'hump' in the packing fraction profiles close to the base that are observed in the experimental results for both $V_{b}{ }^{*}$ values in this limit $(N=700)$, particularly close to the wall. We observe, however, a maximum in the packing fraction profiles closer to the base for relatively large values of $N$ (e.g. when $N=1050)$ which is evident from the $1 \mathrm{D}$ result shown in figure $3(b)$. Also, the temperature profiles (figure 8 ) closer to the wall $\left(r^{*}=11.5\right.$ to 14) show a minimum reflecting the presence of a region of enhanced dissipation. This corresponds closely to the position of enhanced packing fraction described above.

In general, we observe that the viscous models are able to predict the granular temperature and packing fraction profiles reasonably well in a vibrated granular bed. The previously published work by Viswanathan et al. (2006), which compared the results of inviscid models from two closures based on granular kinetic theory, showed the presence of small gradients in pressure across the radius with increase in height. The gradients were, however, significantly less than the pressure gradients in the axial direction of the bed. It was also noted that the effect of increasing the grain-wall inelasticity was to increase the magnitude of the radial pressure gradient. The work pointed out that a non-zero radial pressure gradient results in a net radial force on any given volume element in the cell, which was not balanced by a gravitational body force since gravity acts parallel to the $z$-axis, and physically one would expect such forces to set up convection rolls. This anomalous behaviour is corrected by the use of a full Navier-Stokes-like model developed in the current paper. The inclusion of 

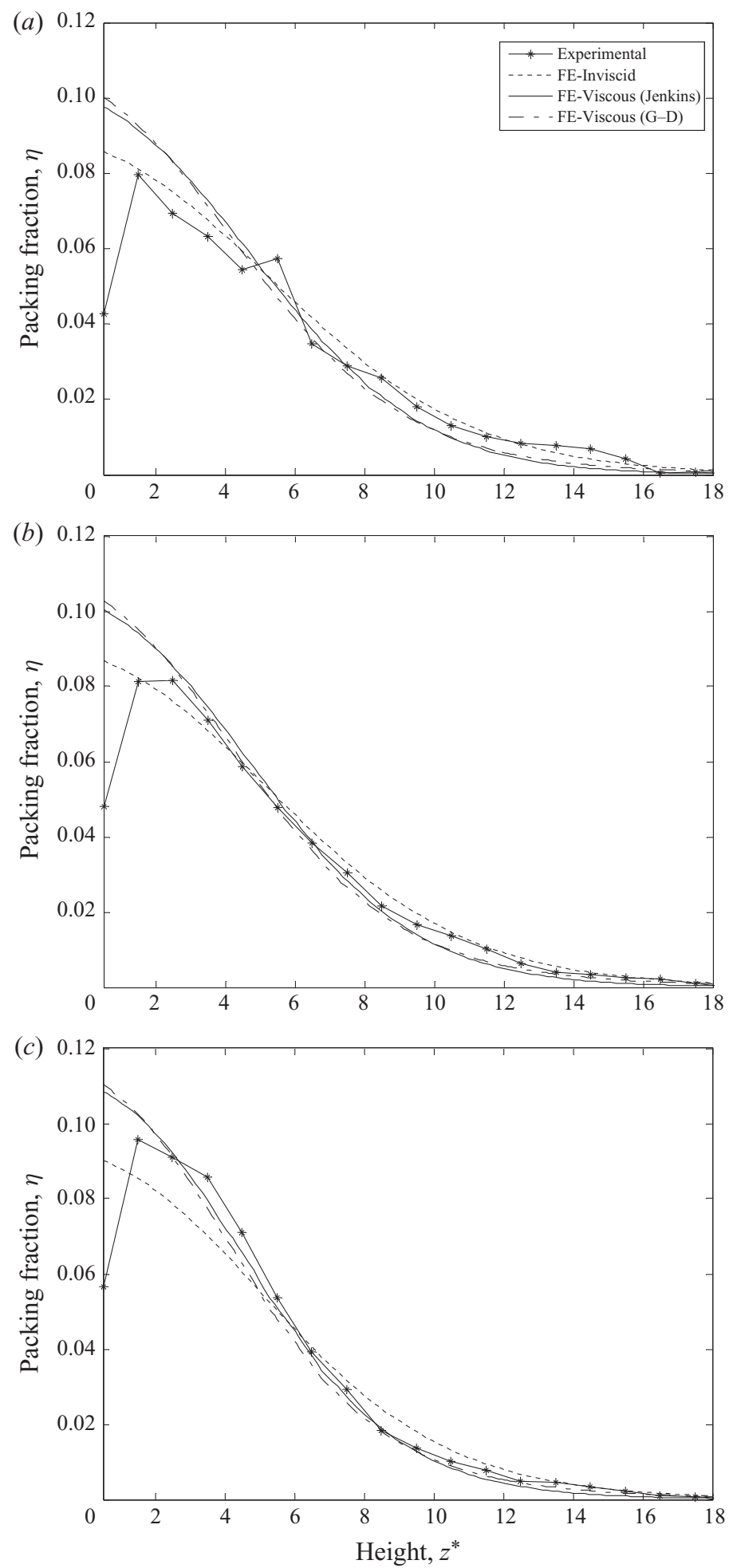

FIGURE 6. Experimental results and predictions from three hydrodynamic models for the vertical cross-sections of packing fraction at the dimensionless radial positions $(a) r^{*}=0.72$, (b) $r^{*}=5.07$ and (c) $r^{*}=9.42$ for the case $e_{w}=0.68, V_{b}^{*}=1.14, N=700$ and $e=e_{b}=0.91$. 

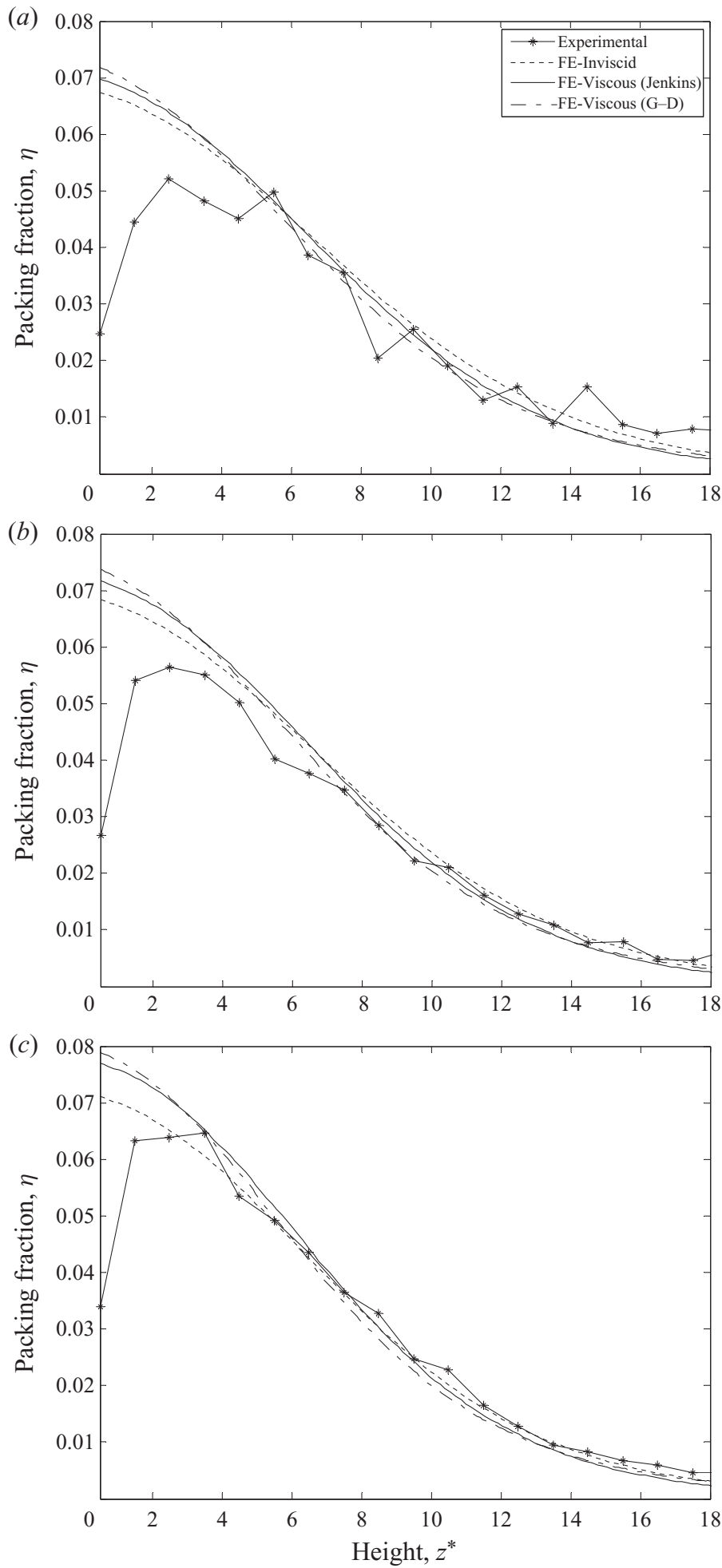

FiguRE 7. Experimental results and predictions from three hydrodynamic models for the vertical cross-sections of packing fraction at the dimensionless radial positions $(a) r^{*}=0.72$, (b) $r^{*}=5.07$ and (c) $r^{*}=9.42$ for the case $e_{w}=0.68, V_{b}^{*}=1.54, N=700$ and $e=e_{b}=0.91$. 


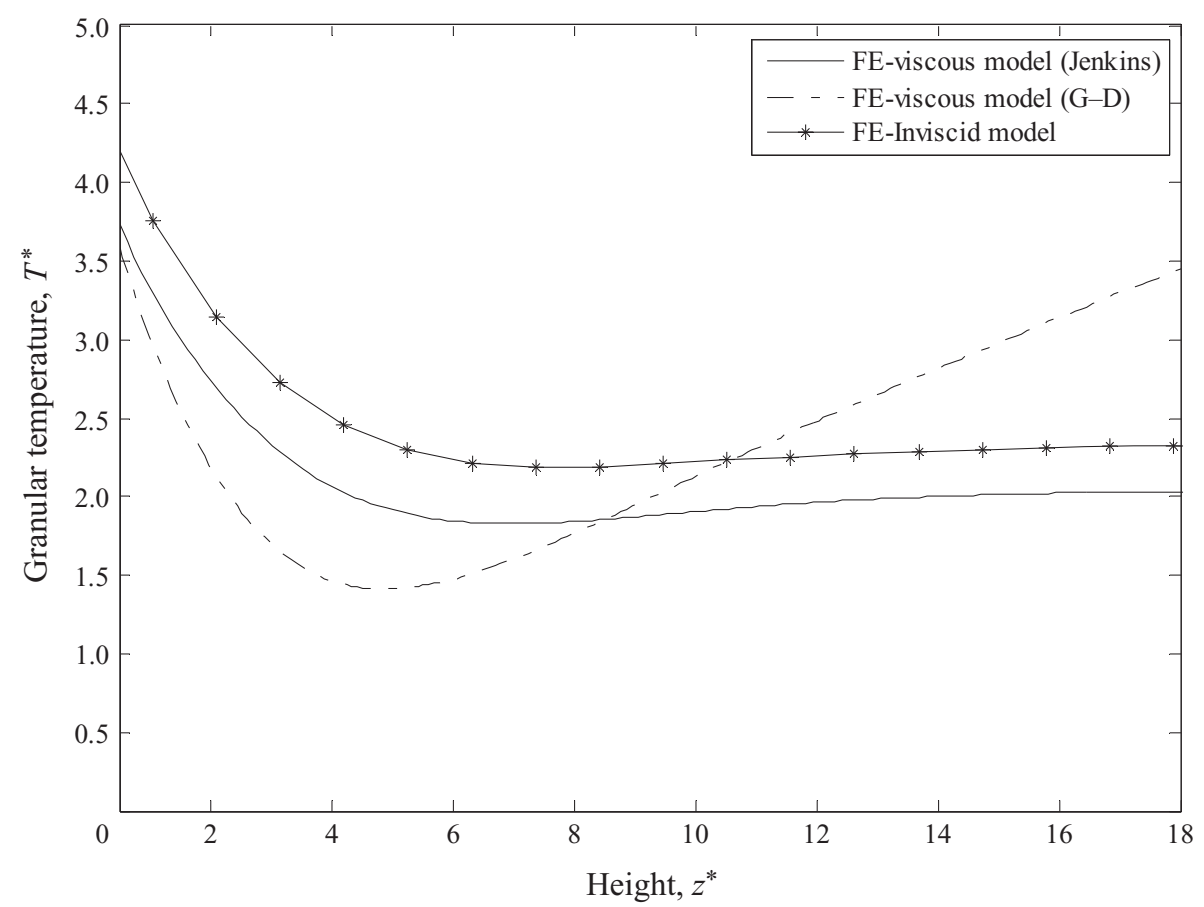

FIGURE 8. Dimensionless granular temperature as a function of altitude predicted by the viscous (Jenkins), viscous $(\mathrm{G}-\mathrm{D})$ and inviscid models for the parameters $r^{*}=13.5, V_{b}^{*}=1.14$, with $e_{w}=0.68, N=1050$ and $e=e_{b}=0.91$.

viscosity allowed us to subsequently compare the convection rolls obtained from the PEPT experiments with those predicted by the model.

Figures 9 and 10 compare the dimensionless mean velocity fields from the viscous models and the experiments in the $\left(r^{*}, z^{*}\right)$ plane for the $V_{b}^{*}$ values discussed above. An asymmetric toroidal convection roll is clearly present in both, where the particles flow, on average, up at the centre and down at the sidewall. For the lower vibration amplitude, the model results (figure $9 a$ and $9 c$ ) predict the 'eye' of the convection roll to be at $r^{*}, z^{*}$ coordinates $\sim(10.2,3.2)$ whereas the experimental results (figure $9 b$ ) show that the roll is centred at $\sim(10.1,4.2)$. When the amplitude of vibration is increased (figure 10) the bed expands and the roll is seen to be centred at a larger height compared to that predicted with $V_{b}^{*}=1.14$ in figure 9 . The centre of the roll predicted by the viscous models (figures $10 a$ and $10 c)$ is $\sim(10,4.4)$ and by the experimental result (figure $10 b)$ is $\sim(10,6.2)$. This shows that the model is able to capture the qualitative features of the convection rolls reasonably well and there is qualitative agreement (in the convection speed) with the experimental results for both vibration amplitudes. The molecular dynamic simulation performed by Talbot \& Viot (2002) for similar parameters showed a good comparison with the PEPT data similar to the agreement that is provided by the hydrodynamic viscous model results. Talbot \& Viot (2002) also performed simulations that demonstrated that the convection rolls of the form observed experimentally can be attributed to wall dissipation. We performed a similar test within our model where the particle-particle coefficient of restitution $(e)$ was set to unity and all the other parameters remained unchanged, and a further test with elastic particle-base collisions. In both cases, the convection rolls are observed (not shown), again demonstrating the role of the wall inelasticity in driving the 

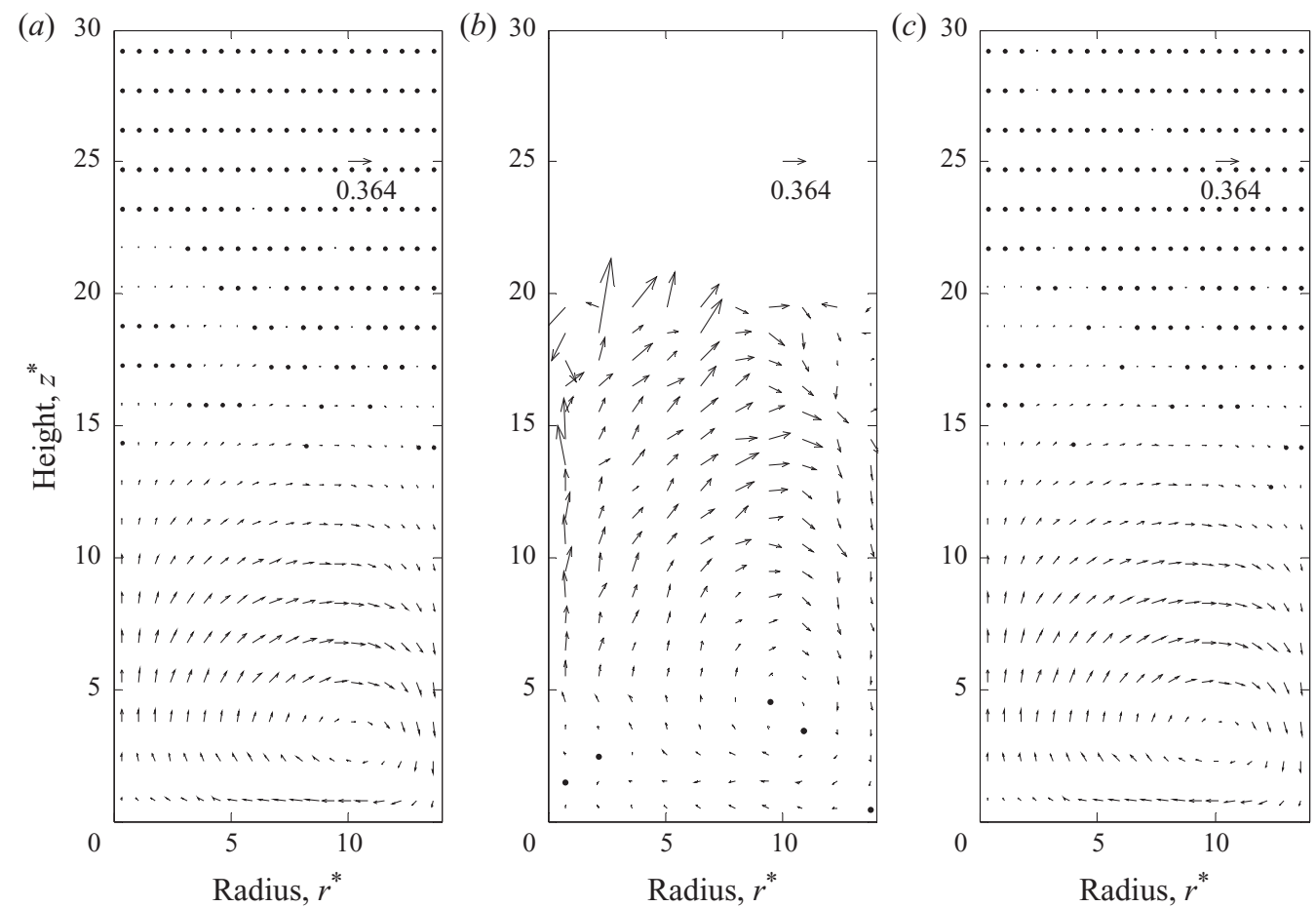

Figure 9. Comparison of dimensionless mean velocity fields predicted by $(a)$ the viscous (Jenkins) model $(b)$ experiment and $(c)$ the viscous $(\mathrm{G}-\mathrm{D})$ model for $V_{b}^{*}=1.14$, with $e_{w}=0.68$, $N=700$ and $e=e_{b}=0.91$.

convection in our system. There are indications that multiple rolls or rolls persistent in the azimuthal direction may become apparent (Sunthar \& Kumaran 2001), but an investigation of these phenomena is out of the scope of this paper.

Figures 11 and 12 show the radial cross-sections of the $r$ - and $z$-components of the mean velocity field at different heights for $V_{b}^{*}=1.14$ and 1.54 , respectively. At relatively lower altitudes, there is a qualitative agreement between the models and the experimental results. We find, however, that the agreement is less good at higher altitudes of the bed. The experimental results show a greater magnitude of the mean velocity fields at larger heights whereas the models predict that the mean velocities are near to zero. From the equations for bulk and shear viscosities $((2.15),(2.16)$ and (2.23), (2.24)), one would expect the expressions to tend to a constant at relatively large altitudes as in this region, the number of particles is low, and the differences between the experiment and the model are largest at the highest altitudes, but the data here are sparse and there is an expectation that the continuum approach might fail here in any case due to the infrequent inter-particle collisions. Also, one must note that, experimentally, the difference in the granular temperatures in different directions cannot be neglected when compared to the model predictions, suggesting that temperature anisotropy should be incorporated to produce a more complete description of the system.

The PEPT experimental data used here from the previously published results (Wildman et al. 2001a,b) have provided evidence to suggest that the convection rolls were caused by buoyancy effects initiated by the enhanced dissipation at the sidewall. As pointed out in $\S 5.1$, numerical solutions were obtained with a particle-sidewall 

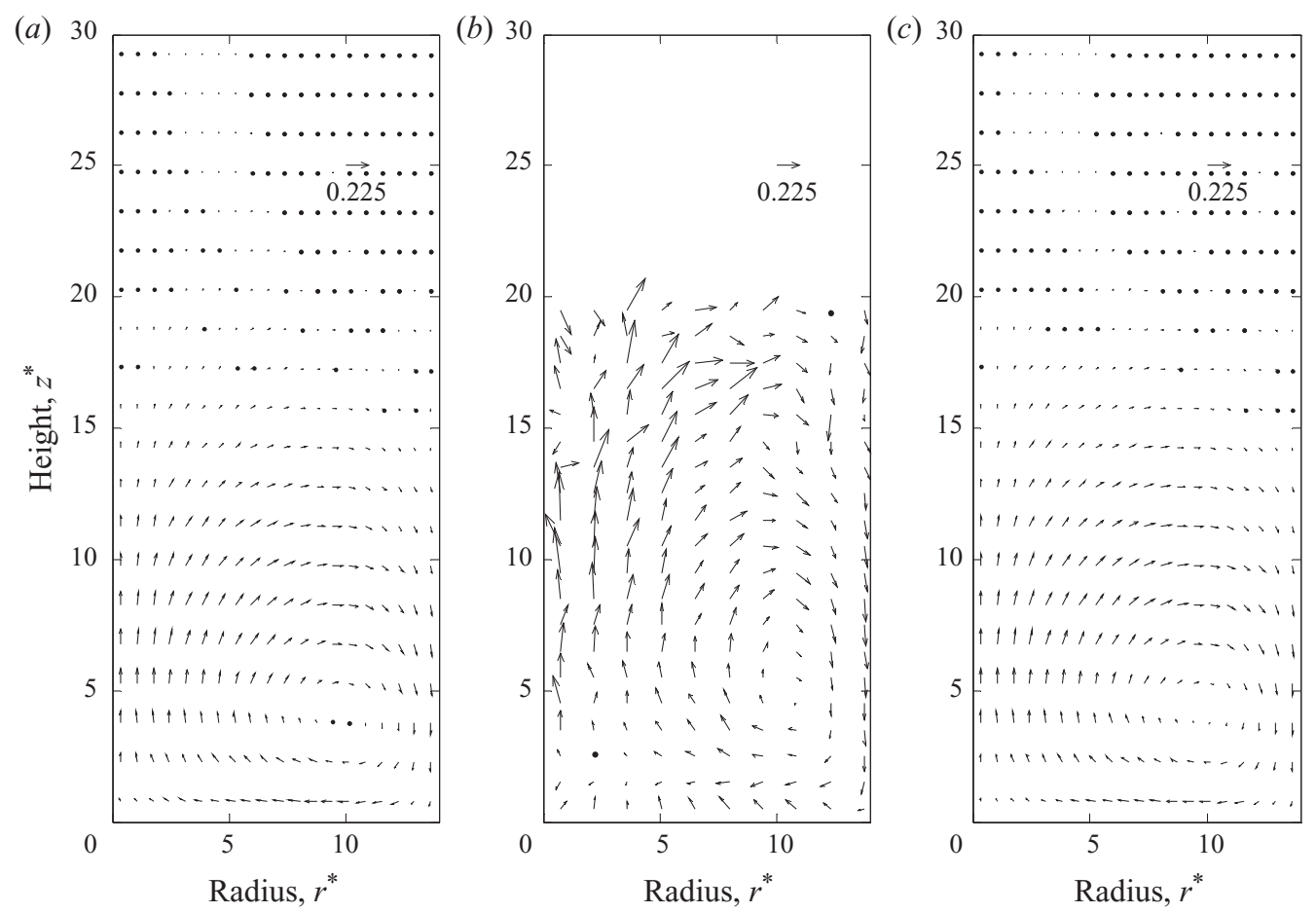

FIGURE 10. Comparison of dimensionless mean velocity fields predicted by $(a)$ the viscous (Jenkins) model $(b)$ experiment and $(c)$ the viscous $(\mathrm{G}-\mathrm{D})$ model for $V_{b}^{*}=1.54$, with $e_{w}=0.68$, $N=700$ and $e=e_{b}=0.91$.

coefficient of restitution $e_{b}=1$, with other parameters unchanged, in order to highlight the influence of the sidewall on convection rolls. No convection was apparent, with the magnitude of the velocity vectors of the order of $10^{-6}$, as mentioned above. However it should be noted that the MD simulations from Talbot \& Viot (2002) showed the presence of some convection in the opposite direction with weak magnitudes of velocity vectors for a similar situation, whereas such a feature is not observed here.

In figure 13 , the dimensionless angular velocity $\left(\Omega^{*}\right)$ (i.e. one half of the vorticity) at the centre of the convection roll obtained from the model is plotted against the dimensionless amplitude $\left(A_{0}^{*}\right)$ for various values of $N$. The magnitude of the angular velocity is seen to decrease with amplitude and appears to approach zero asymptotically for all values of $N$ investigated. This is quite different to the behaviour seen experimentally, where no dependence on amplitude could be observed within the parameter ranges available, but the general trend with $N$ is captured by the simulation, with absolute agreement becoming closer with increasing $N$. The convection rolls are primarily driven by the higher density of material at the wall compared to that in the bulk, which leads to a circulating flow. To investigate this further, we show in figure 14, differences between packing fraction at the wall $\left(\eta_{\text {wall }}\right)$ and that at the axial region $\left(\eta_{\text {axial }}\right)$ as a function of altitude for two different amplitudes $\left(A_{0}^{*}=0.228\right.$, and $A_{0}^{*}=0.548$ ) and for various values of $N, 350$ (figure 14a), 700 (figure 14b), 1050 (figure 14c). These figures demonstrate that the increase in vibration amplitude results in a decrease in the packing fraction difference. It is also observed that increasing the dimensionless base amplitude decreases the radial $\left(r^{*}\right.$, figure $\left.15 a\right)$ and increases the axial $\left(z^{*}\right.$, figure $\left.15 b\right)$ positions of the centre of the convection roll. Bearing in 

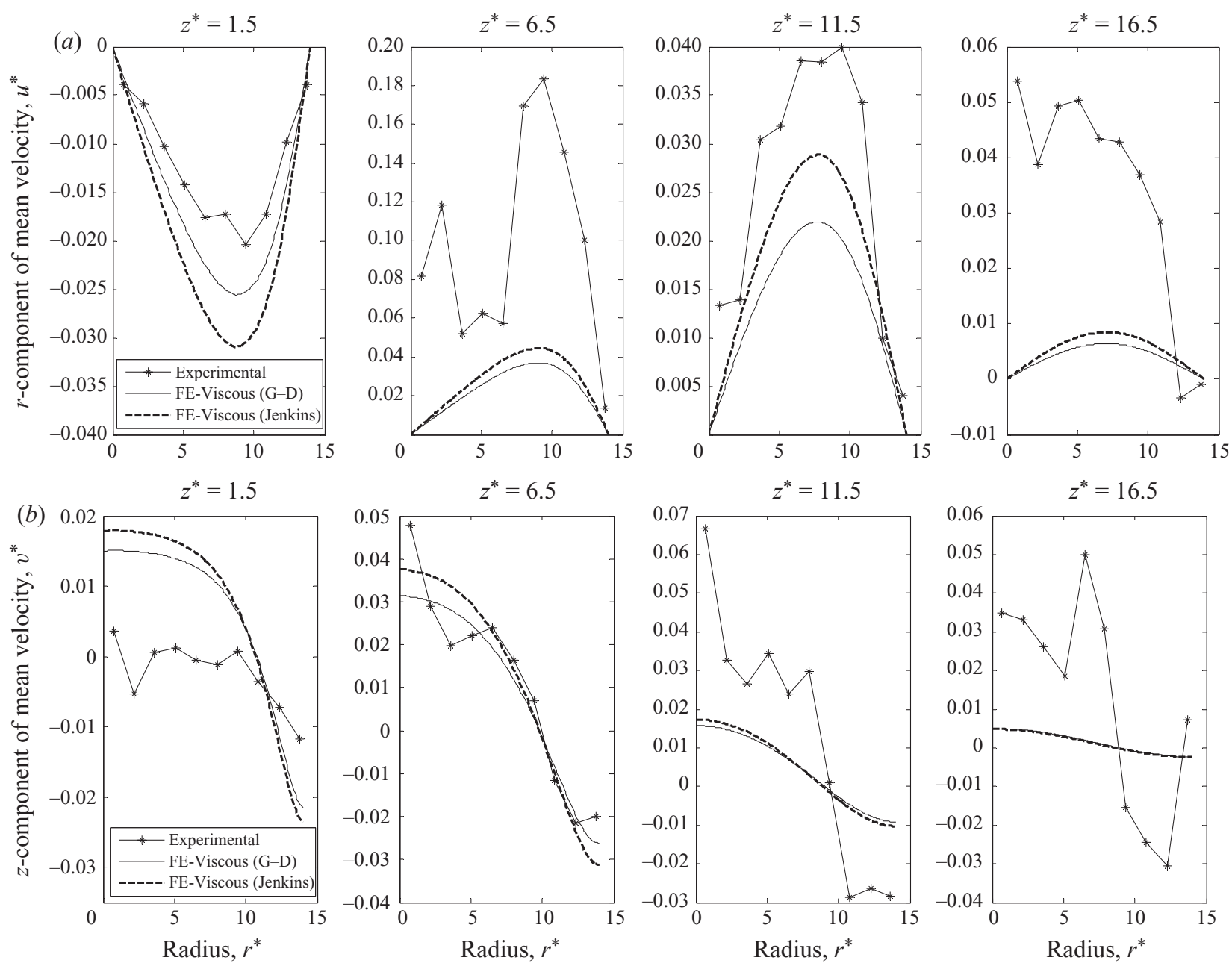

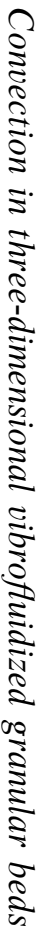

FIGURE 11. Radial cross-sections of the dimensionless $r$-component of the mean velocity field $u^{*}(a)$ and $z$-component of the mean velocity field $v^{*}(b)$ from experiment and two viscous models at different dimensionless heights for $V_{b}^{*}=1.14$, with $e_{w}=0.68, N=700$ and $e=e_{b}=0.91$. 

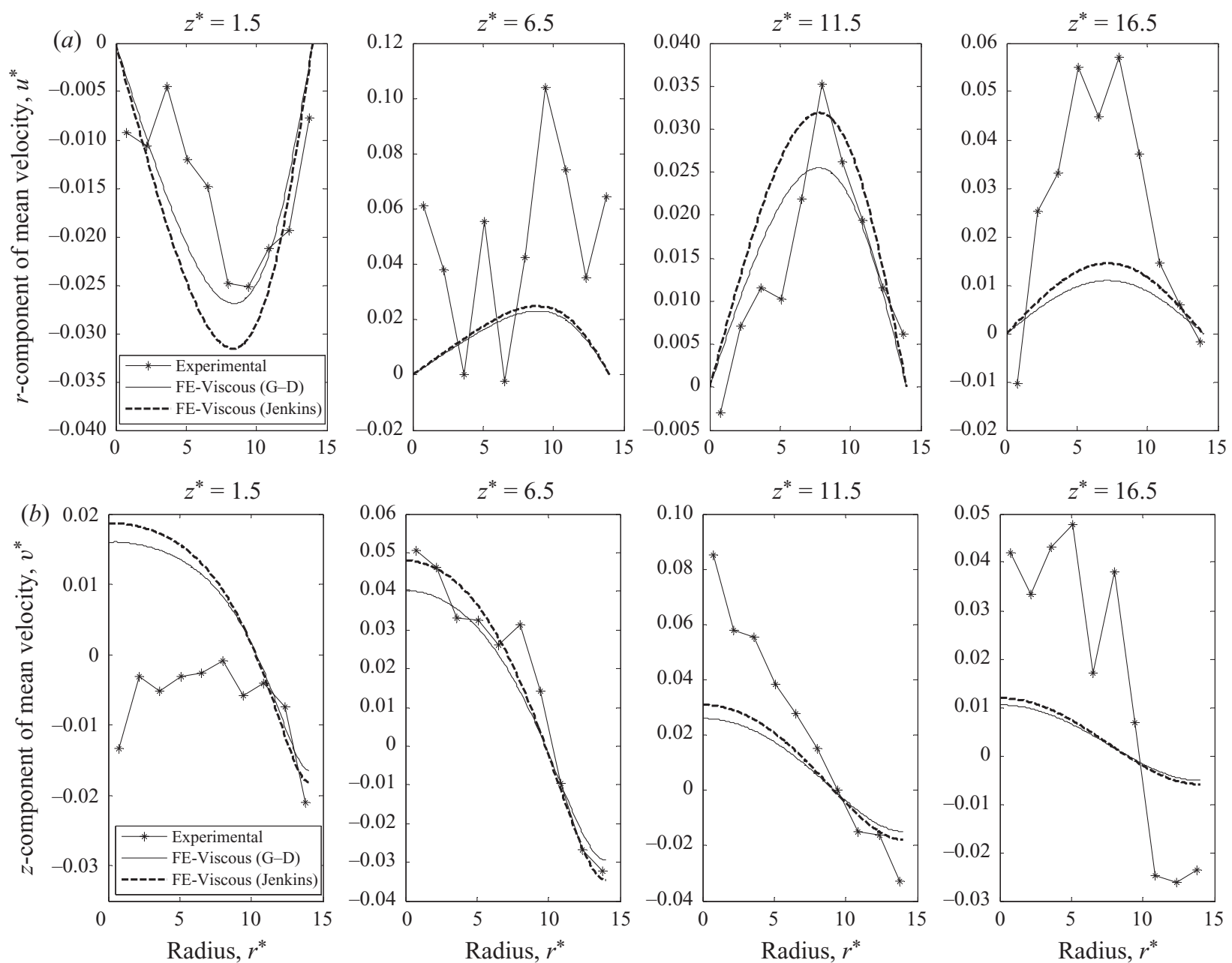

2

FIGURE 12. Radial cross-sections of the dimensionless $r$-component of the mean velocity field $u^{*}(a)$ and $z$-component of the mean velocity field $v^{*}(b)$ from experiment and two viscous models at different dimensionless heights for $V_{b}^{*}=1.54$, with $e_{w}=0.68, N=700$ and $e=e_{b}=0.91$. 


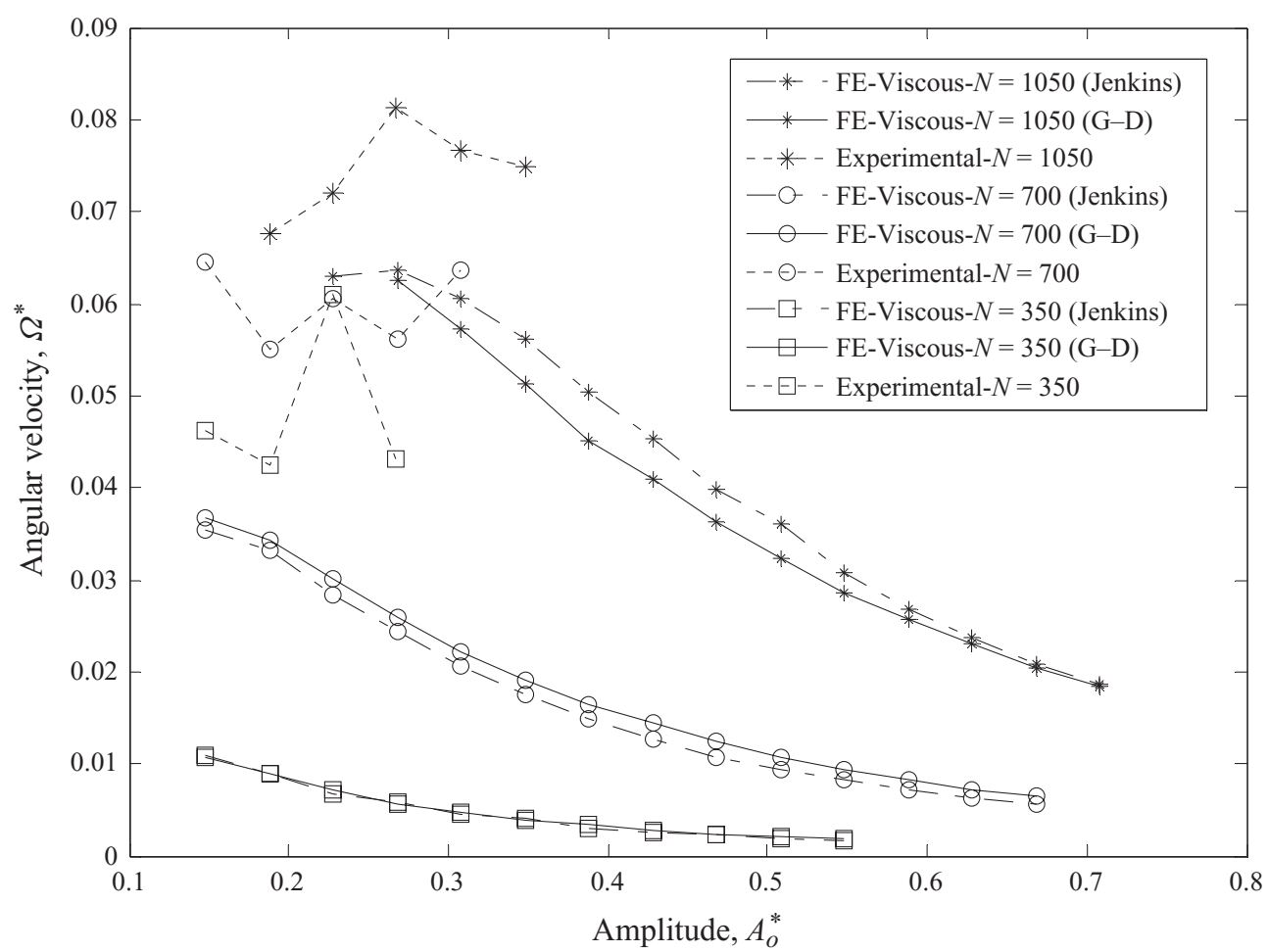

FIGURE 13. The viscous models predictions and experimental values for dimensionless angular velocity of the convection rolls as a function of the amplitude of vibration.

mind the fact that convection is controlled by the dissipation in the region near the wall, it appears that there are several possible causes for the discrepancies between experiment and theory. Firstly, it has already been recognized that there exists a Knudsen layer near a boundary that penetrates several mean free paths into the bulk (Galvin, Hrenya \& Wildman 2007) the absolute size of which depends on the degree of excitation, and within which the forms of the constitutive relations for bulk properties are not always appropriate. Secondly, the model assumes a coefficient of restitution that is velocity-independent. In reality, one anticipates that the coefficient of restitution will reduce with increasing collisional velocities. In terms of the vibrated bed, we would expect more vigorous particle-wall collisions as the amplitude is increased, thus leading to a lower effective coefficient of restitution than that employed, resulting in higher kinetic energy dissipation, higher densities and more convection than predicted, i.e. one would anticipate that the dependence of the angular velocity on the amplitude would be weaker than that predicted.

In spite of the highlighted differences, the agreement between experiment and the model is reasonable, considering the simplifications in a number of potentially important ways (for example, assumptions of isotropic temperature distributions, no rotational motion, etc.). Modifications and improvements to the model could, in principle, be incorporated within the overall solution method outlined in this paper with the introduction of spin granular temperature and particle friction. 

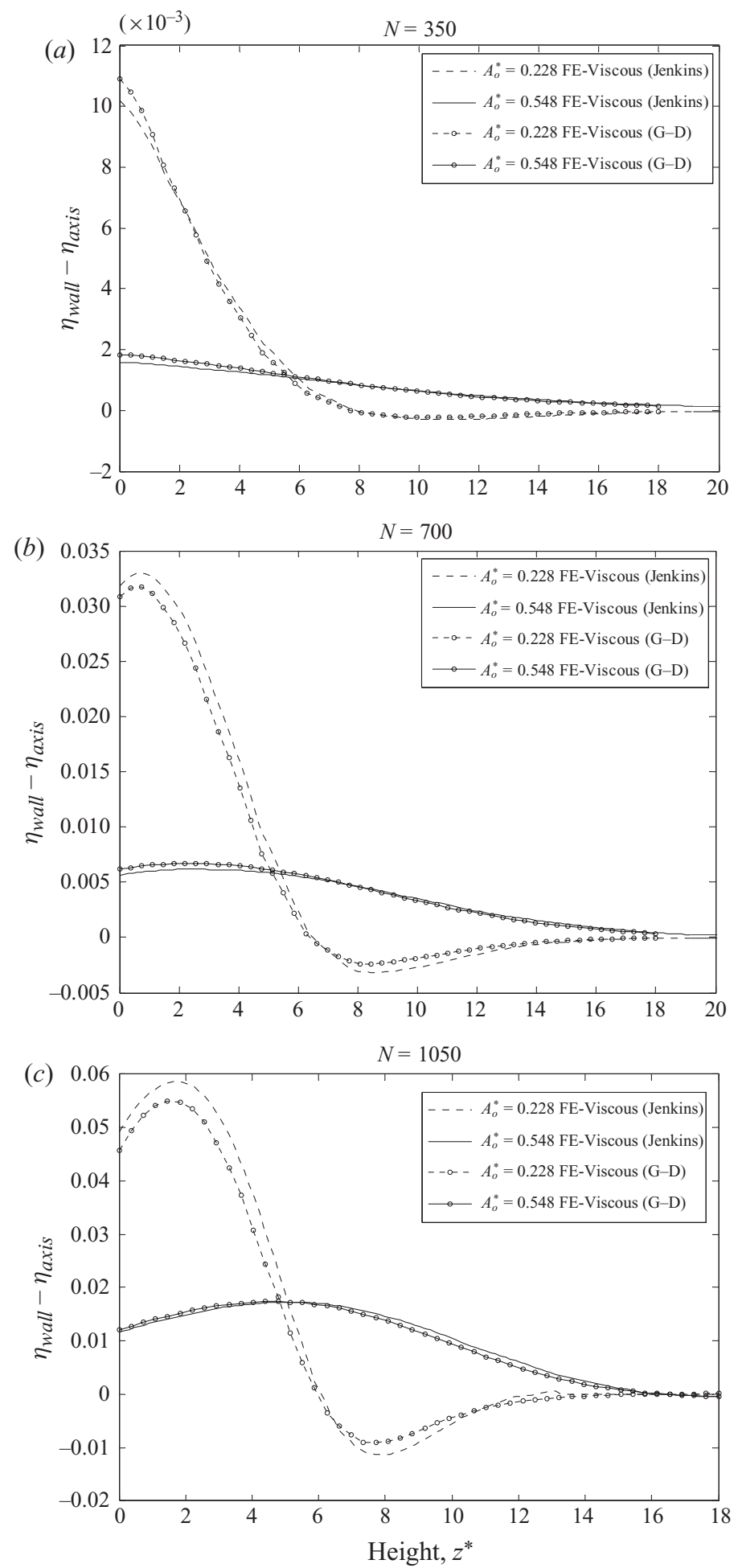

FIGURE 14. Predictions from both viscous models for differences between packing fraction at the wall $\left(\eta_{\text {wall }}\right)$ and axial region $\left(\eta_{\text {axial }}\right)$ of the bed plotted against height $z^{*}$ for $(a) N=350$, (b) 700 and $(c) 1050$ for two different amplitudes, $A_{0}^{*}=0.226$, and $A_{0}^{*}=0.548$ using $e_{w}=0.68$, and $e=e_{b}=0.91$. 

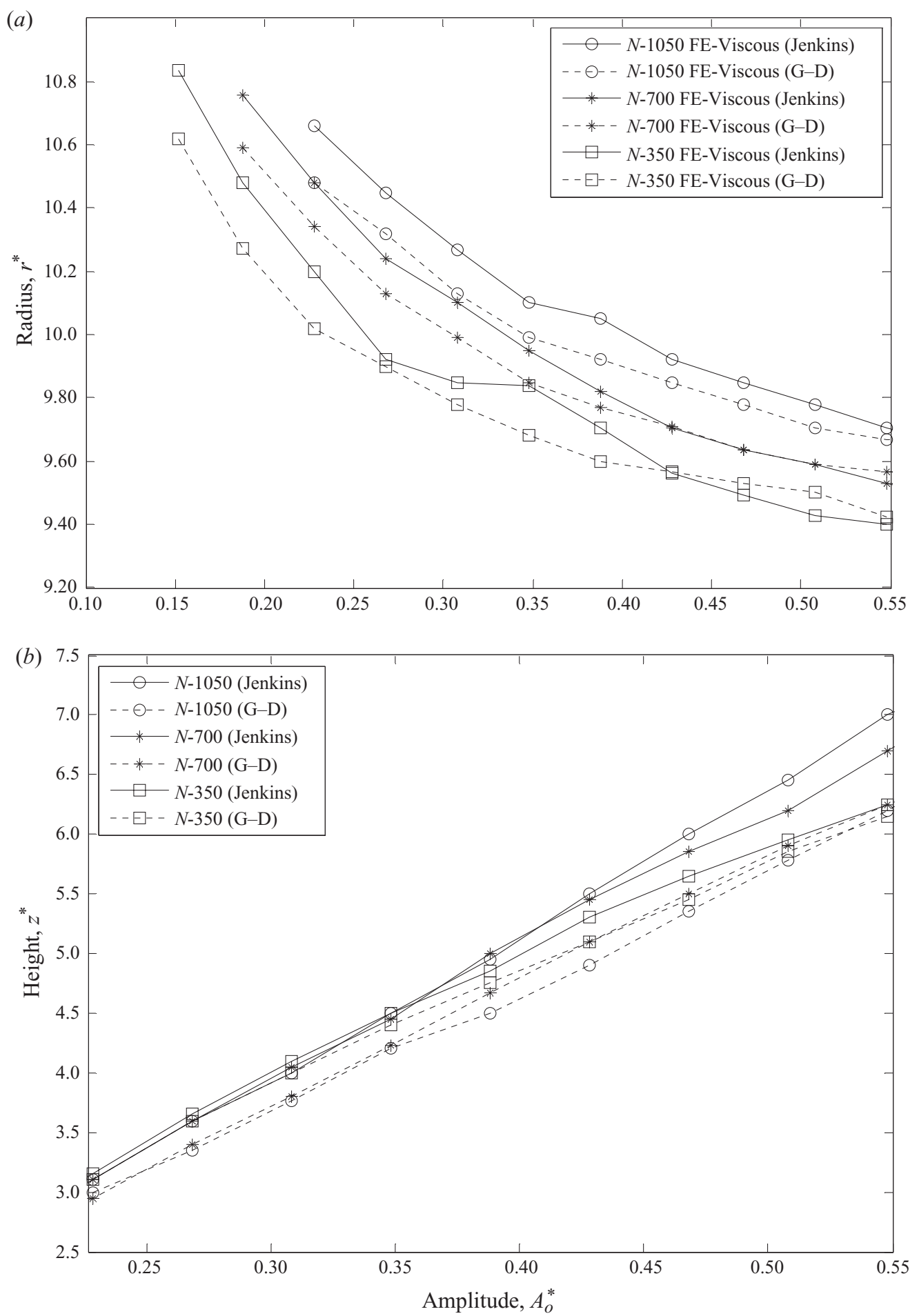

FIGURE 15. The viscous models predictions of dimensionless $(a)$ radial and $(b)$ axial positions of the centre of the convection rolls plotted against $A_{0}^{*}$ for different values of $N$. 


\section{Conclusions}

Viscous hydrodynamic equations resulting from kinetic theory have been solved using the finite-element method in a three-dimensional vibrated monodisperse granular bed and compared to the results of Viswanathan et al. (2006) and experimental results obtained using PEPT. The granular temperature and the packing fraction profiles from the viscous model agree reasonably well with previously obtained inviscid model results and experimental measurements at low altitudes, although it is noted that the large differences in the granular temperatures in different directions are not accounted for in these models. The results of our analysis show reasonable correspondence with experimental results (Wildman et al. 2001a) and MD studies (Talbot \& Viot 2002) of convection in vibro-fluidized granular beds. The velocities of the convection rolls, although small compared to the magnitude of the velocity fluctuations $(\sim 5 \%)$, are broadly in agreement with the experimental results from Wildman et al. (2001a) at relatively low altitudes, but there are discrepancies between the two which may be attributable to the presence of a Knudsen layer and anisotropy in the stress tensor, both of which phenomena require a Burnettlevel description (Hrenya 2008, Sela \& Goldhirsch 1998). In addition, our analysis provides an opportunity for comparison between two different constitutive relations. It was observed that the predicted density profiles for both models were close to one another. There were differences in both the granular temperature and the mean velocity distributions at higher altitudes where the density was lower, but this is a region where the applicability of both models becomes difficult to justify in any case. The Jenkins model provided a closer overall agreement with the experimentally observed total granular temperature profiles, whereas the Garzo-Dufty model tended to match the vertical temperature component better. In the comparison with the experimental mean velocity distributions, neither model was consistently better than the other over the range of experimental parameters studied here.

We thank Dr C. R. Coggrave of Phase Vision Ltd for providing MATLAB image visualization functions. J.M.H. is grateful to the Royal Society and Wolfson Foundation for a Royal Society - Wolfson Research Merit Award. The project was also financially supported by the Engineering and Physical Sciences Research Council through research grant GR/N34208/01. R.D.W. performed the work as part of the EPSRC Advanced Research Fellowship scheme (GR/R75694/01). Some of the research was undertaken during the Granular Physics programme at the Kavli Institute of Theoretical Physics, University of California at Santa Barbara, supported in part by the National Science Foundation under grant number PHY99-07949.

\section{REFERENCES}

Bourzutschky, M. \& Miller, J. 1995 Granular convection in a vibrated fluid. Phys. Rev. Lett. 74, 2216-2219.

Brey, J. J., Dufty, J. W., Kim, C. S. \& Santos, A. 1998 Hydrodynamics for granular flow at low density. Phys. Rev. E 58, 4638-4653.

Carnahan, N. F. \& Starling, K. E. 1969 Equation of state for nonattracting rigid spheres. J. Chem. Phys. 51, 635-636.

Chandrashekar, S. 1981 Hydrodynamic and Hydromagnetic Stability. Dover Publications.

Cordero, P., Ramírez, R. \& Risso, D. 2003 Buoyancy driven convection and hysteresis in granular gases: numerical solution. Physica A 327, 82-87.

Eshuis, P., van Der Meer, D. \& Alam, M. 2010 Onset of convection in strongly shaken granular matter. Phys. Rev. Lett. 104, 038001. 
Eshuis, P., van der Weele, K., van Der Meer, D. \& Lohse, D. 2005 Granular Leidenfrost effect: Experiment and theory of floating clusters. Phys. Rev. Lett. 95, 258001.

Falcon, E., Wunenburger, R., Evesque, P., Fauve, S., Chabot, C., Garrabos, Y. \& Beysens, D. 1999. Cluster formation in a granular medium fluidized by vibrations in low gravity. Phys. Rev. Lett. 83, 440-443.

FARADAY, M. 1831 On a peculiar class of acoustical figures; and on certain forms assumed by groups of particles upon vibrating elastic surfaces. Phil. Trans. R. Soc. Lond. 52, 299-318.

Feitosa, K. \& MenON, N. 2002 Breakdown of energy equipartition in a 2D binary vibrated granular gas. Phys. Rev. Lett. 88, 198301.

Galvin, J. E., Hrenya, C. M. \& Wildman, R. D. 2007 On the role of the Knudsen layer in rapid granular flows. J. Fluid Mech. 585, 73-92.

Garzó, V. \& Dufty, J. W. 1999 Dense fluid transport for inelastic hard spheres. Phys. Rev. E 59, $5895-5911$.

Getling, A. V. 1998 Rayleigh-Benard Convection: Structures and Dynamics, Advanced Series in Nonlinear Dynamics, vol. 11. pp. 13-14. World Scientific Publishing Co., Inc.

Hayakawa, H., Yue, S. \& Hong D. C. 1995 Hydrodynamic description of granular convection. Phys. Rev. Lett. 75, 2328-2331.

Hong, D. C. \& Yue, S. 1998 Traffic equations and granular convection. Phys. Rev. E 58, 4763-4775.

Hrenya, C. M., Galvin, J. E. \& Wildman R. D. 2008 Evidence of higher-order effects in thermally driven rapid granular flows. J. Fluid Mech. 598, 429-450.

Jenkins, J. T. 1999 Kinetic theory for nearly elastic spheres. In Physics of Dry Granular Media, pp. 353-370. Kluwer.

Jenkins, J. T. \& Richman, M. W. 1985 Grad's 13-moment system for a dense gas of inelasticspheres. Arch. Rat. Mech. Anal. 87, 355-377.

Jenkins, J. T. \& Savage, S. B. 1983 A theory for the rapid flow of identical, smooth, nearly elastic, spherical particles. J. Fluid Mech. 130, 187-202.

Khain, E. \& MeErson, B. 2003 Onset of thermal convection in a horizontal layer of granular gas. Phys. Rev. E 67, 021306.

Knight, J. B., Ehrichs, E. E., Kuperman, V., Flint, J. K., Jaeger, H. M. \& Nagel, S. R. 1996 Experimental study of granular convection. Phys. Rev. E 54, 5726-5738.

Kumaran, V. 1998 Temperature of a granular material "fluidized" by external vibrations. Phys. Rev. E 57, 5660-5664.

Landau, L. D. \& Lifshitz, E. M. 1987 Fluid Mechanics, 2nd edn., In Course of Theoretical Physics, vol. 6. Pergamon Press.

Leidenfrost, J. G. 1966 On fixation of water in diverse fire. Intl J. Heat Mass Transfer 9, 1153.

Martin, T. W., Huntley, J. M. \& Wildman, R. D. 2005 Hydrodynamic model for a vibrofluidized granular bed. J. Fluid Mech. 535, 1153-1166.

Meerson, B., Poschel, T. \& Bromberg Y. 2003 Close-packed floating clusters: Granular hydrodynamics beyond the freezing point. Phys. Rev. Lett. 91, 024301.

Paolotti, D., Barrat, A., Marconi, U. M. B. \& Puglisi, A. 2004 Thermal convection in monodisperse and bidisperse granular gases: a simulation study. Phys. Rev. E 69, 061304.

Ramírez, R., Risso, D. \& Cordero, P. 2000 Thermal convection in fluidised granular systems. Phys. Rev. Lett. 85, 1230-1233.

Richman, M. W. 1993 Boundary-conditions for granular flows at randomly fluctuating bumpy boundaries. Mech. Mater. 16, 211-218.

SAVAGE, S. B. 1988 Streaming motions in a bed of vibrationally fluidized dry granular material. J. Fluid Mech. 194, 457-478.

Sela, N. \& Goldhirsch, I. 1998 Hydrodynamic equations for rapid flows of smooth inelasticspheres, to Burnett order. J. Fluid Mech. 361, 41-74.

Shinbrot, T., Khakhar, D., McCarthy, J. \& Ottino, J. M. 1997 A simple model for granular convection. Phys. Rev. Lett. 79, 829-832.

Sunthar, P. \& Kumaran, V. 2001 Characterization of the stationary states of a dilute vibrofluidized bed. Phys. Rev. E 64, 041303.

Tацвот, J. \& Viот, P. 2002 Wall-enhanced convection in vibrofluidized granular systems. Phys. Rev. Lett. 89, 064301. 
Viswanathan, H., Wildman, R. D., Huntley, J. M. \& Martin, T. W. 2006 Comparison of kinetic theory predictions with experimental results for a vibrated three-dimensional granular bed. Phys. Fluids 18, 113302.

WARR, S. \& Huntley, J. M. 1995 Energy input and scaling laws for a single particle vibrating in one-dimension. Phys. Rev. E 52, 5596-5601.

Wildman, R. D., Huntley, J. M., Hansen, J. P., Parker, D. J. \& Allen, D. A. 2000 Single-particle motion in three-dimensional vibrofluidized granular beds. Phys. Rev. E 62, 3826-3835.

Wildman, R. D., Huntley, J. M. \& Parker, D. J. $2001 a$ Convection in highly fluidized threedimensional granular beds. Phys. Rev. Lett. 86, 3304-3307.

Wildman, R. D., Huntley, J. M. \& Parker, D. J. $2001 b$ Granular temperature profiles in threedimensional vibrofluidized granular beds. Phys. Rev. E 6306, 061311.

Wildman, R. D., Martin, T. W., Krouskop, P. E., Talbot, J., Huntley, J. M. \& Parker, D. J. 2005 Convection in vibrated annular granular beds. Phys. Rev. E 71, 061301.

Wildman, R. D. \& Parker, D. J. 2002 Coexistence of two granular temperatures in binary vibrofluidized beds. Phys. Rev. Lett. 88, 064301. 